\title{
Review on numerical modeling of active magnetic regenerators for room temperature applications
}

Nielsen, Kaspar Kirstein; Tusek, Jaka; Engelbrecht, Kurt; Schopfer, Sandro; Kitanovski, Andrej; Bahl, Christian Robert Haffenden; Smith, Anders; Pryds, Nini; Poredos, Alojz

Published in:

International Journal of Refrigeration

Link to article, DOI:

10.1016/j.jirefrig.2010.12.026

Publication date:

2011

Link back to DTU Orbit

Citation (APA):

Nielsen, K. K., Tusek, J., Engelbrecht, K., Schopfer, S., Kitanovski, A., Bahl, C. R. H., Smith, A., Pryds, N., \& Poredos, A. (2011). Review on numerical modeling of active magnetic regenerators for room temperature applications. International Journal of Refrigeration, 34(3), 603-616. https://doi.org/10.1016/j.ijrefrig.2010.12.026

\section{General rights}

Copyright and moral rights for the publications made accessible in the public portal are retained by the authors and/or other copyright owners and it is a condition of accessing publications that users recognise and abide by the legal requirements associated with these rights.

- Users may download and print one copy of any publication from the public portal for the purpose of private study or research.

- You may not further distribute the material or use it for any profit-making activity or commercial gain

- You may freely distribute the URL identifying the publication in the public portal 
Elsevier Editorial System(tm) for International Journal of Refrigeration Manuscript Draft

Manuscript Number: JIJR-D-10-00230R2

Title: Review on numerical modeling of active magnetic regenerators for room temperature applications

Article Type: Review Paper

Keywords: Magnetic refrigerator; Gadolinium; Regeneration; Modelling

Corresponding Author: Mr Kaspar Kirstein Nielsen, M.Sc

Corresponding Author's Institution: Technical University of Denmark

First Author: Kaspar Kirstein Nielsen, M.Sc

Order of Authors: Kaspar Kirstein Nielsen, M.Sc; Jaka Tusek, MSc; Kurt Engelbrecht, PhD; Sandro Schopfer, MSc; Andrej Kitanovski, PhD; Christian Bahl, PhD; Anders Smith, PhD; Nini Pryds, PhD; Alojz Poredos, PhD

Abstract: The active magnetic regenerator (AMR) is an alternative refrigeration cycle with a potential gain of energy efficiency compared to conventional refrigeration techniques. The AMR poses a complex problem of heat transfer, fluid dynamics and magnetic fields, which requires detailed and robust modeling. This paper reviews the existing numerical modeling of room temperature AMR to date. The governing equations, implementation of the magnetocaloric effect (MCE), fluid flow and magnetic field profiles, thermal conduction etc. are discussed in detail as is their impact on the AMR cycle. Flow channeling effects, hysteresis, thermal losses and demagnetizing fields are discussed and it is concluded that more detailed modeling of these phenomena is required to obtain a better understanding of the AMR cycle.

Response to Reviewers: Dear Dr. Ziegler,

We have increased the font size in Fig. 5 of the manuscript according to your requirements. We gratefully thank you for accepting the manuscript and wish you a merry Christmas.

On behalf of the authors,

Kaspar K. Nielsen, PhD. 
1

2

3

\title{
Review on numerical modeling of active magnetic regenerators for room temperature applications
}

\author{
K.K. Nielsen ${ }^{\mathrm{a}, \mathrm{b}}$, J. Tusek ${ }^{\mathrm{c}}$, K. Engelbrecht ${ }^{\mathrm{b}}$, S. Schopfer ${ }^{\mathrm{d}}$, A. Kitanovski ${ }^{\mathrm{c}}$, \\ C.R.H. Bahl ${ }^{\mathrm{b}}$, A. Smith ${ }^{\mathrm{b}}$, N. Pryds ${ }^{\mathrm{b}}$, A. Poredos ${ }^{\mathrm{C}}$ \\ ${ }^{a}$ Department of Mechanical Engineering, Technical University of Denmark \\ Building 425, Niels Koppels Alle, DK-2800 Kgs. Lyngby, Denmark \\ ${ }^{b}$ Fuel Cells and Solid State Chemistry Division \\ Risø National Laboratory for Sustainable Energy \\ Technical University of Denmark, Frederiksborgvej 399, DK-4000 Roskilde, Denmark \\ ${ }^{c}$ University of Ljubljana \\ Faculty of Mechanical Engineering \\ Askerceva c. 6, 1000 Ljubljana, Slovenia \\ ${ }^{d}$ University of Victoria \\ Institute of Integrated Energy Systems \\ ELW B126, PO Box 3055 STN CSC \\ Victoria BC, V8W 3P6, Canada
}

\begin{abstract}
The active magnetic regenerator $(\mathrm{AMR})$ is an alternative refrigeration cycle with a potential gain of energy efficiency compared to conventional refrigeration techniques. The AMR poses a complex problem of heat transfer, fluid dynamics and magnetic field, which requires detailed and robust modeling. This paper reviews the existing numerical modeling of room temperature AMR to date. The governing equations, implementation of the magnetocaloric effect (MCE), fluid flow and magnetic field profiles, thermal conduction etc. are discussed in detail as is their impact on the AMR cycle. Flow channeling effects, hysteresis, thermal losses and demagnetizing
\end{abstract}

Email address: e-mail: kaki@risoe.dtu.dk (K.K. Nielsen) 
fields are discussed and it is concluded that more detailed modeling of these phenomena is required to obtain a better understanding of the AMR cycle. Keywords: Magnetic refrigerator, Gadolinium, Regeneration, Modelling 


\section{Nomenclature}

\begin{tabular}{|c|c|}
\hline \multicolumn{2}{|l|}{ Variables } \\
\hline$T$ & Temperature $[\mathrm{K}]$ \\
\hline$T_{C}$ & Curie temperature $[\mathrm{K}]$ \\
\hline$T_{\infty}$ & Ambient temperature $[\mathrm{K}]$ \\
\hline$\Delta T_{\mathrm{ad}}$ & Adiabatic temperature change $[\mathrm{K}]$ \\
\hline $\mathbf{u}=\left(u_{x}, u_{y}, u_{z}\right)$ & Velocity vector $\left[\mathrm{ms}^{-1}\right]$ \\
\hline$A_{\mathrm{HT}}$ & Wetted area per unit cell $\left[\mathrm{m}^{2} \mathrm{~m}^{-3}\right]$ \\
\hline$c$ & Specific heat capacity $\left[\mathrm{Jkg}^{-1} \mathrm{~K}^{-1}\right]$ \\
\hline$\rho$ & Mass density $\left[\mathrm{kgm}^{-3}\right]$ \\
\hline$k$ & Thermal conductivity $\left[\mathrm{Wm}^{-1} \mathrm{~K}^{-1}\right]$ \\
\hline$h$ & Convective heat transfer coefficient $\left[\mathrm{Wm}^{-2} \mathrm{~K}^{-1}\right]$ \\
\hline$\tau_{1}$ & Timing of magnetization part of the AMR cycle $[\mathrm{s}]$ \\
\hline$\tau_{2}$ & Timing of hot blow part of the AMR cycle $[\mathrm{s}]$ \\
\hline$\tau_{3}$ & Timing of demagnetization part of the AMR cycle $[\mathrm{s}]$ \\
\hline$\tau_{4}$ & Timing of cold blow part of the AMR cycle $[\mathrm{s}]$ \\
\hline$\tau_{\text {rel }}$ & Equal to $\tau_{1} / \tau_{2}=\tau_{3} / \tau_{4}[-]$ \\
\hline$\tau_{\text {tot }}$ & Equal to $2\left(\tau_{1}+\tau_{2}\right)[\mathrm{s}]$ \\
\hline$\mu_{0}$ & Vacuum permeability equal to $4 \pi 10^{-7} \mathrm{NA}^{-2}$ \\
\hline$\mu_{0} H$ & Magnetic field $[\mathrm{T}]$ \\
\hline$M$ & Magnetization $\left[\mathrm{Am}^{-1}\right]$ \\
\hline$D_{p}$ & Dispersion coefficient [-] \\
\hline $\mathrm{Pe}$ & Peclet number [-] \\
\hline$d_{\mathrm{p}}$ & Particle diameter $[\mathrm{m}]$ \\
\hline$d_{\mathrm{r}}$ & Regenerator diameter $[\mathrm{m}]$ \\
\hline$L$ & Length $[\mathrm{m}]$ \\
\hline$V$ & Volume $\left[\mathrm{m}^{3}\right]$ \\
\hline$\dot{m}$ & Mass flow rate $\left[\mathrm{kgs}^{-1}\right]$ \\
\hline$f$ & Frequency $[\mathrm{Hz}]$ \\
\hline$\varphi$ & Utilization [-] \\
\hline$\epsilon$ & Porosity [-] \\
\hline$Q_{\mathrm{c}}$ & Cooling power $\left[\mathrm{Wkg}^{-1}\right]$ \\
\hline$\Delta p$ & Pressure drop [Pa] \\
\hline$\mu_{\mathrm{f}}$ & Dynamic viscosity $[\mathrm{Pa} \cdot \mathrm{s}]$ \\
\hline$K(r)$ & Particle bed permeability $\left[\mathrm{m}^{2}\right]$ \\
\hline
\end{tabular}


Abbreviations

AMR

$\mathrm{MCE}$

Active Magnetic Regeneration

$\mathrm{MCM}$

Magnetocaloric effect

MFM

Magnetocaloric material

Mean field model

HHEX

CHEX

Hot heat exchanger

$\mathrm{HTF}$

Cold heat exchanger

COP

Heat transfer fluid

Coefficient of Performance

\begin{tabular}{|ll|}
\hline Sub-and super scripts & \\
$\mathrm{f}$ & Fluid \\
$\mathrm{s}$ & Solid \\
$i$ & Initial \\
$f$ & Final \\
$\mathrm{HT}$ & Heat transfer \\
Cold & Refers to the cold side reservoir \\
Hot & Refers to the hot side reservoir \\
Stat & Static \\
Eff & Effective \\
Appl & Applied \\
\hline
\end{tabular}




\section{Introduction}

For several decades the active magnetic regenerator (AMR) has been a research topic within the magnetic refrigeration community, as it is a potential alternative to vapor compression technology at room temperature. Such an AMR is based on the magnetocaloric effect (MCE), which manifests itself as a temperature change of a magnetocaloric material (MCM) upon adiabatically changing the magnetic field of the material. Since the maximum adiabatic temperature change of any known MCMs is no more than a few degrees in a magnetic field of one tesla (Pecharsky \& Gschneidner, 2006), the regenerative cycle has to be applied in order to create temperature spans comparable to e.g. those of vapor-compression based cooling systems (Barclay, 1983). Recently, a range of experimental AMR devices have been built and a review of these can be found in Gschneidner \& Pecharsky (2008); Yu et al. (2010). In Yu et al. (2003); Engelbrecht et al. (2007b) general reviews of room temperature magnetic refrigeration are given. Although improvements in AMR performance have been realized, there are currently no commercial devices available, and additional technology development is necessary. Therefore, it is critical to understand the fundamental loss mechanisms, performance limits, and optimal design of AMR systems using detailed models.

Since the AMR involves solid state physics, thermodynamics, fluid dynamics and magnetism a broad range of physicical effects influences the performance of such a system. It is therefore quite important to have reliable numerical models such that the performance trends may be mapped out. A range of such models have been made already, however, a comprehensive review of these models is not available at present. This paper provides such 
a review, which not only include a discussion of the various models but also discusses in detail the various components of an AMR model and how they affect the model results.

\subsection{The AMR cycle}

The AMR cycle consists of four processes, which can overlap. First there is magnetization, where the field applied to the solid regenerator material is increased causing a temperature increase. Magnetization is followed by a fluid flow from the cold fluid reservoir to the hot fluid reservoir, rejecting heat to the ambient. During demagnetization the applied field is then reduced causing the temperature of the regenerator solid to drop and, finally, there is fluid flow from the hot reservoir to the cold, and a cooling load is accepted. The flow processes are governed by the same governing equations as for passive regenerators, which have been studied in detail by, for example, Hausen (1983); Dragutinovic \& Baclic (1998); Willmott (1964). The major difference between passive regenerator models and AMR models is the implementation of the MCE and the timing between the magnetic field profile and the fluid flow profile.

Several approaches to the overall AMR modeling are applied. Steadystate models are simple models, which may provide an estimate of the performance in terms of cooling power versus temperature span as a function of e.g. the geometry of the AMR. Time-dependent models provide a more complex description of the AMR. Since the change of the magnetic field and the fluid flow is inherently time dependent and is coupled with heat transfer between a fluid and a solid, these models capture the physics on a more fundamental level. Both types of models are discussed in the following, although 
the emphasis is put on the time-dependent models, which are dominant in the more recent literature. In Section 2 the specifics of these models are discussed in detail. The remainder of this section gives an overview of the overall development of AMR models.

\subsection{Steady-State AMR Models}

There are several time independent models of AMR systems; these models are sometimes referred to as zero-period or steady-state models. The models generally start from an ideal AMR cycle and reduce the performance individually for estimated losses to axial conduction, heat transfer losses, etc. Steady state models are useful for qualitative investigations of AMR cycle characteristics; for example, the evaluation of the magnetocaloric properties of various materials in the context of an AMR cycle or the parametric investigation of the impact of a particular cycle parameter. The major benefit of these steady-state models is their computational efficiency; however, the predictive capability of a steady state model is limited as they are unable to capture interactions between loss mechanisms. Zhang et al. (2000); He et al. (2003); Zhang et al. (1993) and papers by Yan \& Chen (1991, 1992) all present steady state models that can be used to understand the characteristics of various AMR cycle configurations. Shir et al. (2003) use a time independent model to show how magnetic nanocomposites may be used to obtain an ideal magnetic refrigerant, one in which the local adiabatic temperature change is proportional to the local absolute temperature. Rowe \& Barclay (2003) presents a model based on entropy minimization that predicts the ideal MCE along the length of the regenerator bed. The major shortcomings of all steady state models are their approach to capturing the effect 
of material properties and their macroscopic approach to estimating losses.

\subsection{Time Dependent AMR Models}

Researchers at Astronautics Corp. of America have presented the Finite Reduced Period (FRP) model; this AMR model is one-dimensional and time dependent, but it requires that the heat capacity of the entrained fluid in the regenerator be negligible compared to that of the magnetic material (DeGregoria et al., 1990; DeGregoria, 1991). In this limit, the conventional regenerator equations are solved during the flow portions of the cycle and instantaneous temperature changes are imposed at the conclusion of these processes. These temperature changes represent the magnetization and demagnetization processes, which are assumed to occur reversibly and adiabatically. The pumping loss, axial conduction, and dispersion losses are calculated separately and then subtracted from the predicted refrigeration power (Johnson \& Zimm, 1996).

The FRP model has been applied primarily to the design of low temperature AMR systems that use a gas as the heat transfer fluid, as described by Janda et al. (1989), and therefore the assumption of negligible entrained fluid heat capacity is not overly restrictive.

Kirol \& Mills (1984) describe a one-dimensional transient model of a magnetic cycle that assumes perfect regeneration. Smailli \& Chahine (1998) describe a one dimensional transient model in which only the flow processes are considered; the magnetization and demagnetization processes are assumed to happen instantaneously and reversibly. The heat transfer coefficient is assumed to be constant throughout the regenerator, and the impact of axial conduction and entrained heat capacity is not considered. Hu \& Xiao (1995) 
present an analysis of AMR systems that is based on small perturbation theory; a technique that is used for pulse-tube type refrigeration systems, as described by several researchers including Hooijkaas \& Benschop (1999). The governing equations are linearized and the fluctuating parameters are written in complex form, implying a sinusoidal variation of all such quantities.

These models consider regenerator geometries where the heat transfer between the solid and the fluid is described via a Nusselt number, i.e. the physical domain on which the heat transfer takes place is not resolved. Most geometries, such as packed spheres, wire mesh screens etc. make it quite difficult if not impossible to model the physical situation directly. However, a two-dimensional model of a flat plate AMR is described by Petersen et al. (2008b). The model uses a finite element (FEM) approach to solve for fluid flow profiles and temperature gradients in the solid and the liquid. Because of the increased complexity of the model, the computation time is much higher for the two-dimensional model than equivalent one-dimensional models. The geometry is fixed as a flat plate regenerator and modeling other regenerator geometries would require significant modifications to the existing model. See Appendix A for a summary of the published AMR models to date.

The overall goal of an AMR model is to predict the cooling power versus the temperature span, i.e. the difference in temperature between the hot and cold reservoirs. Including the work performed during the AMR cycle the coefficient of performance (COP) is also available. In this way the theoretical performance of an AMR may be mapped out using a numerical model. 


\section{Components in a numerical AMR model}

This section describes the various aspects of an AMR model. These include the basic equations that are solved, how fluid flow and magnetic field profiles are implemented, how the MCE is addressed etc.

\subsection{Basic energy balance equations}

All numerical models of the AMR are based on a mathematical model describing heat transfer in a solid matrix structure, the MCE in the solid due to the changing magnetic field, and the coupling to the convective heat transfer of a fluid. Thus, the most general energy equation for the regenerator solid may be expressed as

$$
\rho_{\mathrm{s}} c_{\mathrm{s}} \frac{\partial T_{\mathrm{s}}}{\partial t}=\nabla \cdot\left(k_{\mathrm{s}} \nabla T_{\mathrm{s}}\right)+\dot{Q}_{\mathrm{MCE}}+\dot{Q}_{\mathrm{loss}}+\dot{Q}_{\mathrm{HT}}
$$

which describes the heat transfer on the macroscopic scale thus taking into account the intrinsic thermal conductivity of the solid. Subscript $\mathrm{s}$ is for solid, the mass density is denoted by $\rho_{\mathrm{s}}$, the specific heat is $c_{\mathrm{s}}$, temperature is $T_{\mathrm{s}}$, time is $t$, thermal conductivity is $k_{\mathrm{s}}$, the MCE term $\dot{Q}_{\mathrm{MCE}}$, irreversible losses are denoted by $\dot{Q}_{\text {loss }}$ and finally the heat transfer between solid and fluid is denoted $\dot{Q}_{\mathrm{HT}}$. In the case of a $1 \mathrm{D}$ model this will be given through a Nusselt-Reynolds correlation whereas for a 2D or 3D model the boundary interface between solid and fluid is usually spatially resolved and the term is thus expressing an internal boundary condition. However, 2D or 3D models may apply Nusselt-Reynolds correlations as well. The energy equation for 
the heat transfer fluid may be written as

$$
\rho_{\mathrm{f}} c_{\mathrm{f}}\left(\frac{\partial T_{\mathrm{f}}}{\partial t}+(\mathbf{u} \cdot \nabla) T_{\mathrm{f}}\right)=\nabla \cdot\left(k_{\mathrm{f}} \nabla T_{\mathrm{f}}\right)+\dot{Q}_{\mathrm{loss}}-\dot{Q}_{\mathrm{HT}}
$$

Here the subscript $\mathrm{f}$ denotes fluid and $\mathbf{u}=\left(u_{x}, u_{y}, u_{z}\right)$ is the fluid velocity vector. The energy balance equations are assumed valid over the length scale of the regenerator.

The problem intrinsically also involves fluid dynamics and thus the NavierStokes equations must also be solved

$$
\begin{aligned}
\frac{\partial \mathbf{u}}{\partial t}+(\mathbf{u} \cdot \nabla) \mathbf{u} & =\frac{\mu_{\mathrm{f}}}{\rho_{\mathrm{f}}} \nabla^{2} \mathbf{u}-\frac{1}{\rho_{\mathrm{f}}} \nabla p \\
\nabla \cdot \mathbf{u} & =0
\end{aligned}
$$

where $\mu_{\mathrm{f}}$ is the dynamic viscosity and $p$ is pressure. Now, Eqs. 3-4 represent a Newtonian incompressible flow. If, e.g., a gas is used as heat transfer fluid (HTF), the compressible Navier-Stokes equations may be necessary. In most cases Eqs. 3-4 are simplified into analytical expressions, which is the case in the 1D and 2D models (e.g. Nielsen et al. (2009a)) or solved numerically (e.g. Petersen et al. (2008b)).

In general, AMR mathematical models include the following assumptions, also used for passive heat regenerator analysis (Shah \& Sekulic, 2003)

- No phase change in the fluid occurs. As long as water with anti-freeze is used as HTF, this is a fully valid assumption.

- The fluid is incompressible and thus no compression/expansion of the fluid and no pressure oscillations occur during the flow periods. Again, 
when a water/anti-freeze HTF is used this is valid.

- No flow leakage or flow bypassing occurs. This is definitely a simplifying assumption. Experimentally it may be very difficult to control flow bypassing properly.

- Heat transfer caused by radiation within the regenerator is negligible compared to the convective and conductive heat transfer. For near room-temperature applications this is a good approximation since very little heat transfer occurs through radiation.

- The solid within the regenerator is uniformly distributed with no edge effects. This is a simplifying assumption that is notoriously difficult to control in experiments.

\subsection{One-dimensional models}

Many AMR models are one-dimensional and thus assume a Nusselt number correlation as a function of the Reynolds number in order to describe the convective heat transfer between the solid and the fluid. Expressing Eqs. 1-2 in one dimension, the equations for the solid and the fluid in the $1 \mathrm{D}$ case can be defined as:

$$
\begin{aligned}
\rho_{\mathrm{s}} c_{\mathrm{s}} \frac{\partial T_{\mathrm{s}}}{\partial t} & =\frac{\partial}{\partial x}\left(k_{\mathrm{s}} \frac{\partial T_{\mathrm{s}}}{\partial x}\right)+\dot{Q}_{\mathrm{MCE}}+\dot{Q}_{\text {loss }}+\dot{Q}_{\mathrm{HT}} \\
\rho_{\mathrm{f}} c_{\mathrm{f}}\left(\frac{\partial T_{\mathrm{f}}}{\partial t}+u_{x} \frac{\partial T_{\mathrm{f}}}{\partial x}\right) & =\frac{\partial}{\partial x}\left(k_{\mathrm{f}} \frac{\partial T_{\mathrm{f}}}{\partial x}\right)+\dot{Q}_{\text {loss }}-\dot{Q}_{\mathrm{HT}}
\end{aligned}
$$




\subsection{Implementation of the heat transfer between the fluid and the solid}

In all 1D models a heat transfer coefficient, $h$, describing the heat transfer between the fluid and the solid must be used. The heat transfer rate can be written as

$$
\dot{Q}_{\mathrm{HT}}(x)=h A_{\mathrm{HT}}\left(T_{\mathrm{s}}(x)-T_{\mathrm{f}}(x)\right)
$$

where the wetted area per unit cell of the solid material is denoted $A_{\mathrm{HT}}$. Perhaps the most crucial parameter in a 1D model is the heat transfer coefficient. This parameter presents a correlation for the convective heat transfer between the solid and the fluid and the most crucial part of the AMR model thus relies on it. In general, correlations for $h$ are presented in literature (Nusselt-Reynolds correlations). However, often the correlations do not cover the total operational range in terms of the Reynolds number and various correlations exist making it difficult to decide which is the "most correct" to use in a given situation. According to Sarlah \& Poredos (2010) a 10 percent higher heat transfer coefficient yields about 4 percent higher temperature span of the AMR.

The equations for the fluid and the solid in 2D models are usually not coupled through a heat transfer coefficient, but rather an internal boundary condition, which defines thermal contact between the fluid and the solid (Petersen et al., 2008b; Nielsen et al., 2009a; Oliveira et al., 2009). As expected, and as was shown in Petersen et al. (2008a) 1D models may in fact yield very similar results to 2D models given certain circumstances; especially when the fluid channels and solid plates are thin and thus the internal thermal gradients perpendicular to the direction of the flow are negligible.

Sarlah \& Poredos (2005) developed a partial 2D model of the AMR based 
on parallel plates. They used a one-dimensional equation for the heat transfer in the fluid and a two-dimensional heat transfer equation for the solid. Thus, they calculated the temperature distribution in the solid (in the flow direction and a perpendicular direction), but they used a correlation for the heat transfer coefficient for the heat transfer between the fluid and solid (very similar to the regular 1D approach) on the form:

$$
\left.k_{\mathrm{s}} \frac{\partial T_{\mathrm{s}}}{\partial y}\right|_{y=H}(x)=h\left(T_{\mathrm{s}}(x, y=H)-T_{\mathrm{f}}(x, y=H)\right)
$$

where the position in the $y$-direction denoted $H$ refers to the contact point between the solid and fluid.

Since 1D models do not directly account for temperature gradients in the solid material, it has been suggested to reduce the heat transfer coefficient between solid and fluid to account for the losses (Jeffreson, 1972; Engelbrecht et al., 2006). Both Engelbrecht (2008) and Sarlah (2008) used a correction factor for the heat transfer coefficient making it into an effective heat transfer coefficient and thus, to a certain extent, took into account the effect of a non-uniform temperature distribution in the solid perpendicular to the flow direction.

\subsection{Two-dimensional models}

Petersen et al. (2008b) were the first to implement a complete 2D model of a parallel-plate based AMR at room temperature. In their model the spatially resolved dimensions are the $x$ - and $y$-directions, i.e. the direction along the flow and the direction perpendicular to the flow and along the thickness of the solid plate. The equations for the solid and fluid used in the 
Petersen et al. 2D model may be written as

$$
\begin{aligned}
\rho_{\mathrm{s}} c_{\mathrm{s}} \frac{\partial T_{\mathrm{s}}}{\partial t} & =k_{\mathrm{s}}\left(\frac{\partial^{2} T_{\mathrm{s}}}{\partial x^{2}}+\frac{\partial^{2} T_{\mathrm{s}}}{\partial y^{2}}\right) \\
\rho_{\mathrm{f}} c_{\mathrm{f}}\left(\frac{\partial T_{\mathrm{f}}}{\partial t}+u \frac{\partial T_{\mathrm{f}}}{\partial x}\right) & =k_{\mathrm{f}}\left(\frac{\partial^{2} T_{\mathrm{f}}}{\partial x^{2}}+\frac{\partial^{2} T_{\mathrm{f}}}{\partial y^{2}}\right) \\
\rho_{\mathrm{f}}\left(\frac{\partial \mathbf{u}}{\partial t}+(\mathbf{u} \cdot \nabla) \mathbf{u}\right) & =\mu_{\mathrm{f}} \nabla^{2} \mathbf{u}-\nabla p \\
\nabla \cdot \mathbf{u} & =0
\end{aligned}
$$

assuming constant thermal conductivity and that $\mathbf{u}=\left(u_{x}, u_{y}, 0\right)$. The heat transfer between the solid and fluid domains is modeled through an internal boundary condition, which can be expressed as

$$
k_{\mathrm{s}} \frac{\partial T_{\mathrm{s}}}{\partial y}=k_{\mathrm{f}} \frac{\partial T_{\mathrm{f}}}{\partial y}
$$

which is valid on the boundary between the two domains only. Oliveira et al. (2009) formulated the 2D AMR problem in a very similar way, albeit using non-dimensional variables.

Very recently, Liu \& Yu (2010) presented a 2D model of a porous structure. The authors show that it is possible to track the 2-dimensional temperature distribution in the regenerator bed. In this way internal temperature gradients orthogonal to the flow direction may be resolved.

The equations presented above (1 and 2) (for both 1D and 2D models) include the effect of thermal conduction in the solid and the fluid, convective heat transfer, viscous losses, heat losses to the surroundings and, of course, the MCE. These effects have varying influence on the operation of an AMR and different models thus include various effects, which are discussed below 
in detail.

\subsection{Three-dimensional models}

Bouchard et al. (2009) presented a three-dimensional model of the AMR with a regenerator comprised of particles of spherical and elliptical nature. Their model solves the fully coupled problem with the governing equations including Eqs. 1-2, the incompressible Navier-Stokes equations and the relevant magnetostatic equations describing the coupling between the applied magnetic field, magnetization and internal magnetic field. The model of Bouchard et al. (2009) is of great interest since it is the first (published) attempt to model the full geometry of an AMR including magnetostatics. Such a model may provide deeper insights into the actual ongoing physics in the regenerator. The results are so far of a limited nature, however, improvements and further results are expected.

\subsection{Other mathematical models}

Kitanovski et al. (2005) developed a numerical steady state model for a rotary AMR. The model was described in cylindrical coordinates. The radial dimension was neglected. Because of the higher frequency the longitudinal heat conduction was neglected as well. Results of the analysis provided a 2D map of temperature gradients in the solid and fluid, respectively.

\subsection{Boundary conditions}

Initial and boundary conditions have to be specified in order for any AMR model to be solved. These conditions include hot and cold side fluid inlet temperatures and boundary conditions towards the ambient. The common way of defining the boundary conditions is given in Tab. A.1. 


\section{TABLE 1}

In the $2 \mathrm{D}$ and $3 \mathrm{D}$ cases an internal boundary condition similar to that given in Eq. 13 is needed to describe heat transfer between the fluid and the solid. Steady state operation is specified by setting the temperature of the fluid and solid at the beginning of the cycle to the temperature at the end of the previous cycle.

\subsection{Implementation of the magnetocaloric effect}

In order to analyze the operation of the AMR, magnetic properties need to be included in the model. The adiabatic temperature change, $\Delta T_{\mathrm{ad}}$, and specific heat of the solid is generally a function of both temperature and magnetic field and appropriate look-up tables should be applied. The MCE is generally implemented in one of two ways.

The simplest and most straightforward way of including the MCE in the model is to apply the adiabatic temperature change to the solid during the processes of magnetization or demagnetization directly. This may be formulated mathematically as

$$
T=T_{i}+\Delta T_{\mathrm{ad}}\left(T_{i}, \mu_{0} H_{i}, \mu_{0} H_{f}\right)
$$

where the initial temperature is denoted $T_{i}$, the initial magnetic field $H_{i}$ and the final magnetic field is $H_{f}$.

The adiabatic temperature change as a function of temperature, initial and final magnetic field can be derived from experimental data tables or using the mean field model (MFM) (Morrish, 1965) and many authors have used the MFM in their AMR numerical models (Petersen et al., 2008b; Nielsen 
et al., 2009a; Smailli \& Chahine, 1998; Li et al., 2006; Allab et al., 2005; Siddikov et al., 2005; Oliveira et al., 2009; Aprea et al., 2009; Tagliafico et al., 2010; Sarlah \& Poredos, 2005; Kitanovski et al., 2005).

The following equation may be used to describe the energy release in the magnetocaloric material during magnetization or demagnetization over a period of time

$$
\dot{Q}_{\mathrm{MCE}}=-T_{\mathrm{s}} \frac{\partial M}{\partial T} \mu_{0} \frac{\partial H}{\partial t}
$$

with the volumetric magnetization denoted $M$. This equation is simply derived from the basic thermodynamics of the MCE using the Maxwell relation between the derivative with respect to magnetic field of the entropy and the derivative of the magnetization with respect to temperature. This expression was employed in the models published by e.g. Shir et al. (2004); Engelbrecht et al. (2007a); Nielsen et al. (2009a). This way of implementing the MCE is a so-called built-in method.

The built-in method for including the MCE in the model presupposes a continuous change of the magnetic field, which will certainly always be the case in an experiment. However, this method requires detailed, and numerically differentiable data sets of the magnetization and specific heat as functions of both temperature and magnetic field. These may not always be available from experimentally obtained data for MCMs.

The processes of magnetization and demagnetization in an AMR can be simulated by both methods. However, the selection of the most suitable method in general depends on the purpose of the simulations. If the main goal of the numerical model is to simulate actual experimental AMRs with high accuracy, it is crucial to use the experimentally obtained magnetocaloric 
properties of the chosen magnetocaloric material. However, in the case that sufficient experimental data is not available, the direct application of the adiabatic temperature change may be the best method of applying the MCE.

\subsection{Effect of longitudinal thermal conduction}

Longitudinal thermal conduction is included in most models. It has a large influence on the operation of the AMR under certain geometric and operational circumstances, especially for regenerators with a relatively short length and a structure continuously connected along the flow direction (e.g.

parallel plates) and/or for small values of the utilization, where the fluid is, of course, moved a short distance. The utilization is defined as the ratio of the thermal mass of the HTF moved to the total thermal mass of the regenerator solid

$$
\varphi=\frac{\dot{m}_{\mathrm{f}} c_{\mathrm{f}} \tau_{2}}{m_{\mathrm{s}} c_{\mathrm{s}}},
$$

where the mass flow rate is denoted $\dot{m}_{\mathrm{f}}$ and the duration of the blow period is $\tau_{2}$. This is also related to the frequency of the operation. A lower frequency means a larger influence of the longitudinal thermal conduction.

Figure A.1 shows the impact of the longitudinal thermal conduction at different mass flow rates and at two different operating frequencies. It should be noted that the thermal conduction is extremely important to consider at low mass flow rates (low utilizations) and low cycle frequency, since under these conditions the convective heat transfer due to fluid movement is of the same order as the thermal conduction of the fluid and does thus not dominate the heat transfer of the fluid as it does for larger mass flow rates.

FIGURE 1 
Among the published AMR numerical models, some include longitudinal thermal conduction in the solid as well as in the fluid (Petersen et al., 2008b; Nielsen et al., 2009a; Kawanami et al., 2006; Siddikov et al., 2005; Tagliafico et al., 2010; Legait et al., 2009; Dikeos et al., 2006), which is physically the most correct. Engelbrecht (2008); Sarlah (2008); Dikeos et al. (2006) included longitudinal thermal conduction in the system through an effective longitudinal thermal conduction. In porous media, such as a packed sphere regenerator, the conduction path through the solid and fluid is complex and difficult to separate and model independently. Therefore, the fluid/solid matrix is modeled as a single entity regarding longitudinal thermal conduction, which is expressed in the parameter $k_{\text {eff }}$. Such a measure not only simplifies the equation for the fluid, but may also improve the stability of the numerical simulation (Sarlah, 2008). The effective longitudinal thermal conduction of the solid and the fluid may be expressed as

$$
k_{\mathrm{eff}}=k_{\mathrm{stat}}+k_{\mathrm{f}} D_{p}(\mathrm{Pe})
$$

where $D_{p}$ is the dispersion coefficient, which is a function of the Peclet number, Pe. Correlations for the static conduction, $k_{\text {stat }}$, and the dispersion coefficient may be found in e.g. Hadley (1986).

Thermal dispersion is a complex phenomenon and may be understood as thermal conduction due to hydrodynamic mixing in the fluid. This mixing occurs due to the geometry of the solid structure and is thus much more complicated to derive in a packed sphere based regenerator than in, e.g., parallel-plate based regenerators. A contiuously connected solid as, e.g., parallel plates may have a significant dispersion due to higher longitudinal 
thermal conductivity.

\subsection{Effect of viscous dissipation}

Viscous dissipation in the fluid is the irreversible degradation of mechanical energy into heat and may have a large impact on the thermal analysis of the regenerator. The impact of the viscous losses is included in most models using a friction factor correlation as presented in e.g. Engelbrecht (2008); Sarlah (2008); Dikeos et al. (2006). Viscous dissipation is generally low for most prototype AMRs and is often neglected in models of AMRs and other regenerators. However, as regenerator geometries reduce in size and AMRs operate at higher frequency, which requires higher fluid flow to maintain an equal utilization, viscous dissipation will increase and may become significant for future AMR configurations or operating conditions.

Figure A.2 shows the impact of the pressure drop on the COP of packed spheres AMR with water as a heat transfer fluid at different mass flow rates. Note that pressure drop (viscous losses) affects the COP through irreversible viscous losses as well as through the work needed to pump the fluid through the AMR. The impact on the COP is seen to be most profound at higher mass flow rates (higher utilizations) as expected.

FIGURE 2

\subsection{Heat losses}

Most AMR models assume perfect insulation to the ambient and ignore thermal interactions with the regenerator housing. That means that parasitic losses due to inevitable temperature gradients between the regenerator 
and the surroundings are neglected. Only one model has included a formulation of the parasitic losses to ambient through the concept of an extra "half" dimension (Nielsen et al., 2009a). This extra spatial dimension is not numerically resolved but a lumped heat loss term is applied and found through analyzing the thermal resistance from the regenerator core to the ambient. Results show that this effect may have a significant impact on the AMR performance (Nielsen et al., 2009a,c). Figure A.3 shows an example of including the thermal losses in a numerical AMR model.

Frischmann et al. (2009) present a model that considers the thermal interaction between the fluid and regenerator housing using a dispersion model that considers radial temperature gradients within the regenerator. Experimental single blow data showed that the regenerator housing significantly reduced the apparent heat transfer in the regenerator, especially at low Reynolds numbers (Frischmann et al., 2009). Thermal interactions with the regenerator housing and with the ambient can be a significant loss mechanism for AMRs. However, the authors are not aware of work that studies these losses in detail.

FIGURE 3

\subsection{Magnetic field change}

In general, the magnetic field change can be distinguished between discrete "on-off" and a continuous change (Fig. A.4). If the discrete magnetic field change is assumed, the inclusion of the MCE is limited to the application of the adiabatic temperature change directly since the built-in method is meaningful only with continuous magnetic field changes. However, if the purpose of the numerical model is to simulate the experimental operation 
of an AMR, it may be important to implement the time-dependent change of the magnetic field as the magnetic field change and fluid flow processes often overlap in real AMR devices. The time-dependent change of the magnetic field can generally be handled with both methods of including the MCE presented in Sec. 2.8.

\section{FIGURE 4}

Most AMR models neglect spatial-dependent magnetic field changes and assume that each piece of magnetocaloric material in the AMR is subject to the same magnetic field change at a given point in time. In Nielsen et al. (2009a) an experimental AMR device was modeled with a spatially resolved applied magnetic field. Bjørk \& Engelbrecht (2011) show that the synchronization and width of the magnetic field can be of great importance to the AMR performance. The effect of the demagnetizing field, presented in Sec. 2.8, may have a strong influence on the spatial variation of the internal magnetic field in an AMR. The demagnetizing field is generally a function of geometry, temperature and the material properties of the MCM (Smith et al., 2010; Brug \& Wolf, 1985).

\subsection{Materials properties}

The physical properties of the fluid and the solid are important to include in a physically realistic way. The heat transfer fluid most commonly assumed when modeling AMRs is water perhaps with added anti-corrosives and anti-freeze (Engelbrecht, 2008; Aprea et al., 2009; Tagliafico et al., 2010; Petersen et al., 2008b; Nielsen et al., 2009a). In this case the fluid may safely be assumed to be incompressible and most authors also assume constant fluid properties, i.e. viscosity, mass density and specific heat (Petersen et al., 
2008b; Nielsen et al., 2009a; Li et al., 2006; Allab et al., 2005; Oliveira et al., 2009; Aprea et al., 2009; Dikeos et al., 2006), whereas a few have implemented models with temperature-dependent properties (Engelbrecht, 2008; Engelbrecht et al., 2007a; Siddikov et al., 2005). When the temperature of water is changed, for example, from 0 to $40{ }^{\circ} \mathrm{C}$ the mass density and specific heat are consequently changed by less than 1 percent, while the dynamic viscosity may depend on temperature but has less effect on the performance of the AMR. If, for example, a gas is used as the heat transfer fluid, the assumption of constant physical properties would lead to a much greater error since mass density, specific heat, thermal conductivity and dynamic viscosity of gasses depend significantly on temperature and pressure. Also, an equation of state is needed if the flow cannot be considered incompressible. However, the effect on the AMR performance due to temperature-dependent fluid properties has not been investigated in great detail yet.

Many authors assume temperature independence of mass density and thermal conductivity of the MCM (see Table A.2). This assumption depends highly on the MCM considered. Considering e.g. gadolinium the thermal conductivity and the mass density do not change significantly around room temperature (see e.g. Jacobsson \& Sundqvist (1989) for details) whereas at both lower and higher temperatures the thermal conductivity is dependent on temperature. The specific heat of the MCM varies significantly with temperature and magnetic field - especially around the magnetic transition temperature of the material - and should thus not be assumed to be constant. Also, some materials (usually exhibiting a $1^{\text {st }}$ order transition) have a structural transition close to the magnetic phase transition temperature. 
This often induces changes in the volume of the material and thus also the mass density and perhaps even the thermal conductivity.

It is important that the thermodynamic MCM properties are consistent. If care is not taken when determining specific heat and the corresponding entropy change with magnetization or adiabatic temperature change, model predictions can become unrealistic. An example of inconsistent thermodynamic properties is the assumption of a specific heat that is independent of magnetic field combined with a constant adiabatic temperature change with magnetization. If the specific heat of the material is used to calculate the entropy curves for zero field and a high magnetic field, the two will be equal because the specific heat is constant. This means that the entropy change with magnetization, and therefore adiabatic temperature change, is zero, which contradicts the assumption of a constant non-zero adiabatic temperature change. Using a material with constant specific heat with an assumed adiabatic temperature change will result in an over prediction of cooling power, and a cycle that does not obey the $2^{\text {nd }}$ law of thermodynamics.

\subsection{Flow conditions}

A periodic fluid flow is present in all numerical AMR models. It is of great importance to implement the fluid flow correctly and several approaches for this have been made. Two main considerations should be done carefully.

- The assumptions about the actual flow include whether the flow is laminar, incompressible, fully developed, temperature dependent etc.

- The representations of the change in input velocity can be a discrete 
step function, following a sinusoidal curve or whichever profile an experimental AMR device uses.

In models where the flow is transversally resolved (in one or two dimensions perpendicular to the flow direction) a flow-profile is needed. If the geometry is simple the profile may be derived analytically as is the case for models of parallel plate regenerators (Nielsen et al., 2009a) or in more advanced cases a numerical solution to the Navier-Stokes equation for the fluid velocity profile may be needed (Bouchard et al., 2009).

The determination of the mean fluid velocity is usually done through a fixed mass flow rate or similar; however, the temporal change of the mean fluid velocity is implemented differently. Some authors assume a discrete velocity profile as a function of time such that the flow is either on or off (Li et al., 2006; Allab et al., 2005; Siddikov et al., 2005; Aprea et al., 2009; Petersen et al., 2008a; Nielsen et al., 2009a), perhaps through a ramping method (Petersen et al., 2008b; Nielsen et al., 2009a) and some models assume a more realistic contiuous flow curve as a function of time (Dikeos et al., 2006; Nielsen et al., 2009a; Engelbrecht, 2008; Oliveira et al., 2009). It was argued in Nielsen et al. (2010) that for the general purpose of theoretical evaluation of the AMR performance discrete velocity profiles may be the best option since it removes the possible impact of specific experimental devices. In Nielsen et al. (2009a) and Nielsen et al. (2010) it was argued that when modeling experimental devices it is of great importance to actually make the flow profile in the numerical model resemble that of the experiment, which may seem obvious but is not necessarily always how models are implemented. 


\subsection{Channeling effects}

Flow channelling is caused by a non uniform porosity distribution in the transverse bed direction. For a packed particle bed the porosity at the wall is typically greater than the porosity at the center position of the regenerator. As a consequence, the pore velocity near the wall will be larger than the center velocity due to the lower pressure drop close to the wall (Kaviany, 1995; Achenbach, 1995). Flow channelling will result in cold or hot bypasses that will lower the effectiveness of the regenerator (Chang \& Chen, 1998). The amount of flow channeling depends greatly on the ratio of regenerator diameter, $d_{\mathrm{r}}$, to particle diameter, $d_{\mathrm{p}}$. The flow channeling becomes more important with decreasing ratio $d_{\mathrm{r}} / d_{\mathrm{p}}$ (Nemec $\&$ Levec, 2005). In order to resolve the radial velocity distribution the volume averaged transport equations for the momentum transport may be used (Hsu, 2005).

$$
\epsilon(r) \frac{\mathrm{d} p}{\mathrm{~d} z}=\mu\left(\frac{\mathrm{d}^{2} u_{z}}{\mathrm{~d} r^{2}}+\frac{1}{r} \frac{\mathrm{d} u_{z}}{\mathrm{~d} r}\right)-\frac{\mu u_{z}}{K(r)}-F \rho \frac{\left|u_{z}\right| u_{z}}{\sqrt{K(r)}}
$$

Here $u_{z}$ is understood as the superficial velocity, i.e. the velocity the flow would have if the bed was empty, in the axial direction. The permeability for a particle bed is $K=\epsilon^{3} d_{\mathrm{p}}^{2} /\left(a(1-\epsilon)^{2}\right)$ and the Forchheimer factor $F=b / \sqrt{a \epsilon^{3}}$ with $a=150, b=1.75$ and $\epsilon$ being the porosity. In this sense the regenerator is understood as a continuum described by a radial porosity distribution. An extensive review on porosity distributions for packed beds can be found in du Toit (2008). They strongly recommend the use of the following correlation for the porosity

$$
\epsilon(r)=\epsilon_{\infty}+\left(1-\epsilon_{\infty}\right) \exp \left[-\frac{N}{d_{p}} r\right]
$$


with $N=6000$ and $\epsilon_{\infty}=V_{\mathrm{f}} /\left(V_{\mathrm{f}}+V_{\mathrm{s}}\right)$ being the bulk porosity. Equation (18) can be solved with standard solvers in, e.g., Matlab using the boundary conditions $\mathrm{d} v_{z}(r=0) / \mathrm{d} r=0$ and $v_{z}(r=R)=0$. The pressure gradient is assumed to be constant (i.e. obtained from experiments).

FIGURE 5

Figure A.5 shows the radial velocity profile close to the wall. A significant departure of the radial velocity adjacent to the wall from the center velocity is observable. There are two ways to resolve flow channeling in an actual model for a magnetic refrigerator device: resolve the regenerator on a 2D computational domain or account for a modified pressure drop and heat transfer correlation that takes flow channeling (and therewith the ratio $d_{\mathrm{r}} / d_{\mathrm{p}}$ ) into account (Achenbach, 1995). So far the channeling effect has not been studied in detail in terms of its impact on the AMR cycle. This may certainly pose a significant issue to address.

\subsection{Modeling of graded AMRs}

It has been experimentally shown that grading the regenerator along the flow direction with a range of MCMs each with a different Curie temperature increases the AMR performance (Rowe \& Tura, 2006). This is an area of the magnetic refrigeration research where numerical models may prove to have the most significant impact. The optimal performance of the AMR as a function of multiple MCMs, i.e. through a variation of the Curie temperatures of each material, the number of materials and perhaps even the amount of each material, pose a very large problem due to the many free parameters. In this area only a few models have been applied (Jacobs, 2009; Engelbrecht et al., 2007b; Nielsen et al., 2009b) and further work to understand the grading 
effect is urgently needed. Layered regenerators are generally modeled by assigning solid material properties as a function of position in the regenerator. Several problems arise when considering the modeling of graded regenerators. Apart from the vast parameter space, magnetocaloric data for each of the individual materials may not yet be available to such a degree that it is usable for this kind of modeling. Also, the interface between each material should be considered. This could demand spatially varying thermal conductivity, mass density etc. It is noted that knowledge of whether the amount of each individual MCM should be the same for optimal performance of the AMR or if it could be beneficial to have an asymmetrical distribution of the materials. The definition of the problem inherently also includes the intended application. Figure A.6 shows a schematic of the concept of layering an AMR bed.

FIGURE 6

\subsection{Implementing the effect of demagnetization}

It is well-known that the internal magnetic field of a magnetic material in a homogeneously applied magnetic field can be highly inhomogeneous, an effect known as geometric demagnetization (Bouchard et al., 2005, 2009; Smith et al., 2010; Joseph \& Schloemann, 1965; Brug \& Wolf, 1985; Peksoy \& Rowe, 2005). In fact, the internal magnetic field may be reduced to as little as a few percent of the applied field dependent on the temperature of the sample, the sample's geometry, and direction and magnitude of the applied magnetic field (Smith et al., 2010). This effect may be understood through

the demagnetizing field, which is generally dependent on the geometry of the magnetic material and the orientation of the applied magnetic field as 
well as the spatially non-constant magnetization, which is a function of both the internal magnetic field and temperature in turn. This emphasizes the highly non-linear nature of the demagnetization problem and it is basically impossible to simplify it into e.g. an extra source term in the energy equation of the solid. A fully coupled numerical model for calculating the internal magnetic field is thus needed and should be solved simultaneously with the heat transfer model.

It is emphasized that the MCE, whether expressed as the isothermal entropy change or the adiabatic temperature change, should be considered as a function of the internal magnetic field. Of course, measurements may be reported as a function of applied magnetic field, but in order to compare materials properties of different materials between different experimental setups the internal magnetic field is the proper independent variable (and, of course, so is also the temperature).

So far only a few published numerical AMR models have included this effect (Bouchard et al., 2005, 2009; Nielsen et al., 2010; Peksoy \& Rowe, 2005). In Bouchard et al. $(2005,2009)$ the effect of demagnetization was included as an extra coupled equation to be solved together with the thermal equations. However, the results were not discussed in detail in terms of the impact of this on the AMR cycle. It was shown, however, that the adiabatic temperature change may be considerably affected when accounting for demagnetization (Bouchard et al., 2005), which is consistent with the recent results from Christensen et al. (2010) and Bahl \& Nielsen (2009).

In Peksoy \& Rowe (2005) the demagnetization was investigated for a symmetric regenerator setup and the resulting magnetization showed as a 
function of position in the regenerator under various conditions. The results showed that care should indeed be taken when deciding how to align the applied magnetic field with respect to the regenerator material when considering thermal gradients in the system etc.

In other extreme cases, such as described in Bahl \& Nielsen (2009), the effect may be significant. An example of the resulting internal magnetic field is shown in Fig. A.7. It is apparent that there is a vast difference between the resulting internal magnetic field dependent on the orientation of the applied magnetic field and the temperature of the MCM. The more ferromagnetic the material is the more significant the effect is. In the case of applying the field perpendicular to the largest surface of the plate (Fig. A.7b) the internal field may be decreased with up to 80 percent for the cases considered here.

\section{FIGURE 7}

\subsection{Hysteresis effect in AMR modeling}

In literature it is often argued that with a $1^{\text {st }}$ order magnetic transition MCMs are among the most promising candidates as refrigerants in an AMR device due to their large MCE. However, at least three very important aspects of this assumption have not yet to our knowledge been investigated in detail. Firstly, the MCE is usually confined to a quite narrow temperature interval for $1^{\text {st }}$ order materials compared to $2^{\text {nd }}$ order materials. Secondly, the specific heat usually has a high but narrow peak around the Curie temperature and the peak temperature changes as a function of magnetic field (e.g. Palacios et al. (2010)). Thirdly, the inherent hysteretic effects present in most $1^{\text {st }}$ order materials (e.g. Pecharsky \& Gschneidner (2006) and Tocado et al. (2009)) have not yet been considered in any published AMR model. 
In Basso et al. $(2005,2006)$ the fundamentals of hysteresis were considered for magnetic materials and to some extent that analysis covered initial steps to evaluate the impact on the AMR cycle. In Kitanovski \& Egolf (2009) the hysteretic losses were implemented as a scalar quantity expressing a degradation of the efficiency of an AMR device. However, this efficiency was estimated and not found through a rigorous analysis. At present the hysteresis effect has not been implemented in any AMR model. Generally, an analysis of the impact of the special behavior of the magnetocaloric properties of $1^{\text {st }}$ order materials should certainly be performed. The operating frequency of the AMR cycle may be limited by e.g. the inherently slower $1^{\text {st }}$ order transition (Gschneidner et al., 2005). See Kuz'min (2007) for other examples of limiting factors to the AMR frequency.

\section{Conclusion}

A large range of numerical AMR models were discussed. The individual components of a general AMR model were described in detail and their impacts were discussed. The rank, or dimensionality, of the individual AMR models ranges from 1D to 3D. Most models published are 1D of nature and thus include a heat transfer correlation to describe the heat transfer between the solid regenerator matrix and the heat transfer fluid. It was also argued, on the other hand, that 2- or 3D models are difficult to realistically implement to model complex structures different from e.g. parallel plates, even though a first attempt of full 3D-modeling of a particle bed has been published. It is therefore concluded that each kind of model is relevant to consider and that

the requirements of the particular case modeled should be carefully analyzed 
when choosing which kind of model to use.

The various components of an AMR model, such as the implementation of the MCE, flow profiles etc., were discussed in detail. It may generally be concluded that it is important to ensure that the $2^{\text {nd }}$ law of thermodynamics is not violated. Furthermore, each component should be implemented as detailed as possible, which includes the use of proper experimental data, consideration of the resulting internal magnetic field, proper applied magnetic field and flow profiles in accordance with any experiment modeled etc. It should be stressed, however, that simpler models are usually much easier to interpret and, especially, to ensure to be numerically well-behaved. It may therefore be recommended to use a simple model to try to identify the most important physical processes of a given geometry and configuration, and to build on that to implement more sophisticated models.

The modeling of AMR cannot be said to be sufficient as is. Several very interesting physical aspects have not been considered yet, at least not in detail. The hysteresis inherent in most $1^{\text {st }}$ order materials should be the topic of detailed future investigations as should the special specific heat curves that such materials exhibit. The effect of demagnetization on the performance of the AMR should also be the topic of detailed future investigations.

\title{
Appendix A. Summary of published AMR models
}

\author{
TABLE A.1
}




\section{Acknowledgements}

K.K. Nielsen, K. Engelbrecht, C.R.H. Bahl, A. Smith and N. Pryds thank the support of the Programme Commission on Energy and Environment (EnMi) (Contract no. 2104-06-0032) which is part of the Danish Council for Strategic Research. K. K. Nielsen also thanks the Danish Agency for Science, Technology and Innovation under the Danish Ministry for Science, Technology and Innovation.

\section{References}

\section{References}

Achenbach, E. (1995). Heat and flow characteristics of packed beds. Experimental Thermal and Fluid Science, 10(1):17-27.

Allab, F., Kedous-Lebouc, A., Fournier, J., and Yonnet, J. (2005). Numerical modeling for active magnetic regenerative refrigeration. IEEE Transactions on Magnetics, 41(10):3757-3759.

Aprea, C., Greco, A., and Maiorino, A. (2009). A numerical analysis of an active magnetic regenerative cascade system. In Egolf, P. W., editor, Third International Conference on Magnetic Refrigeration at Room Temperature, pages 259-265. International Institute of Refrigeration.

Bahl, C. R. H. and Nielsen, K. K. (2009). The effect of demagnetization on the magnetocaloric properties of gadolinium. Journal of Applied Physics, 105(1):013916 (5 pp.). 
Bahl, C. R. H., Petersen, T. F., Pryds, N., and Smith, A. (2008). A versatile magnetic refrigeration test device. Review of Scientific Instruments, 79(9):093906.

Barclay, J. A. (1983). Theory of an active magnetic regenerative refrigerator. NASA Conference Publication, pages 375-387.

Basso, V., Bertotti, G., LoBue, M., and Sasso, C. (2005). Theoretical approach to the magnetocaloric effect with hysteresis. Journal of Magnetism and Magnetic Materials, 290-291(Part 1):654-657.

Basso, V., Sasso, C. P., Bertotti, G., and LoBue, M. (2006). Effect of material hysteresis in magnetic refrigeration cycles. International Journal of Refrigeration, 29(8):1358-1365.

Bjørk, R. and Engelbrecht, K. (2011). The influence of the magnetic field on the performance of an active magnetic regenerator (AMR). International Journal of Refrigeration, 34:192-203.

Bouchard, J., Nesreddine, H., and Chahine, R. (2005). Impact of demagnetization on magnetocaloric effect in pure gadolinium. 1st International Conference on Magnetic Refrigeration at Room Temperature, pages 93101.

Bouchard, J., Nesreddine, H., and Galanis, N. (2009). Model of a porous regenerator used for magnetic refrigeration at room temperature. International Journal of Heat and Mass Transfer, 52(5-6):1223-1229.

Brug, J. and Wolf, W. (1985). Demagnetizing fields in magnetic measurements i. thin discs. Journal of Applied Physics, 57(10):4685-4694. 
Chang, Z.-C. and Chen, P.-H. (1998). Flow channeling effect on a regenerator's thermal performance. Cryogenics, 38(2):191-196.

Christensen, D. V., Bjørk, R., Nielsen, K. K., Bahl, C. R. H., Smith, A., and Clausen, S. (2010). Spatially resolved measurements of the magnetocaloric effect and the local magnetic field using thermography. Journal of Applied Physics, 108(6):063913.

DeGregoria, A. (1991). Modeling the active magnetic regenerator. Advances in Cryogenic Engineering, 37(pt B):867-873.

DeGregoria, A. J., Barclay, J. A., Claybaker, P. J., Jaeger, S. R., Kral, S. F., Pax, R. A., Rowe, R., and Zimm, C. B. (1990). Preliminary design of a 100 w $1.8 \mathrm{k}$ to $4.7 \mathrm{k}$ regenerative magnetic refrigerator. Advances in Cryogenic Engineering, 35:1125-1131.

Dikeos, J., Rowe, A., and Tura, A. (2006). Numerical Analysis of an Active Magnetic Regenerator (AMR) Refrigeration Cycle. AIP Conference Proceedings, 823(1):993 - 1000.

Dragutinovic, G. D. and Baclic, B. S. (1998). Operation of Counterflow Regenerators. Computational Mechanics Inc., Billerica, MA.

du Toit, C. (2008). Radial variation in porosity in annular packed beds. Nuclear Engineering and Design, 238(11):3073-3079.

Engelbrecht, K. (2008). A Numerical Model of an Active Magnetic Regenerator Refrigerator with Experimental Validation. $\mathrm{PhD}$ thesis, University of Wisconsin, Madison. 
Engelbrecht, K., Nellis, G. F., and Klein, S. A. (2007a). Comparing modeling predictions to experimental data for active magnetic regenerative refrigeraton systems. In Egolf, P. W., editor, Second International Conference on Magnetic Refrigeration at Room Temperature, pages 349-357. International Institute of Refrigeration.

Engelbrecht, K. L., Nellis, G. F., and Klein, S. A. (2006). The effect of internal temperature gradients on regenerator matrix performance. Journal of Heat Transfer, 128(10):1060-1069.

Engelbrecht, K. L., Nellis, G. F., Klein, S. A., and Zimm, C. H. (2007b). Review article: Recent developments in room temperature active magnetic regenerative refrigeration. HVAC and $R$ Research, 13(4):525.

Frischmann, M., Jacobs, S., Nellis, G., and Klein, S. (2009). Measuring nusselt number using a single-blow regenerator facility. In Egolf, P. W., editor, Third International Conference on Magnetic Refrigeration at Room, pages 443-448. International Institute of Refrigeration.

Gschneidner, K A, J., Pecharsky, V. K., and Tsokol, A. O. (2005). Recent developments in magnetocaloric materials. Reports on Progress in Physics, 68(6):1479-1539.

Gschneidner, K. and Pecharsky, V. (2008). Thirty years of near room temperature magnetic cooling: Where we are today and future prospects. International Journal of Refrigeration, 31:945-961.

Hadley, G. R. (1986). Thermal conductivity of packed metal powders. International Journal of Heat and Mass Transfer, 29(6):909-919. 
Hausen, H. (1983). Heat Transfer in Counterflow, Parallel-Flow and Crossflow. McGraw-Hill Co.

He, J., Chen, J., and Wu, C. (2003). The influence of heat-transfer laws on the performance of a magnetic stirling refrigeration cycle. International Journal of Ambient Energy, 24(2):75-82.

Hooijkaas, H. W. G. and Benschop, A. A. J. (1999). Pulse tube development using harmonic. In Ross, R. G., editor, Simulations, Proc. 10th Int. Cryocooler Conf., pages 359-367, New York , NY. Kluwer Academic/Plenum Publishers.

Hsu, C. H. (2005). Dynamic Modeling of Convective Heat Transfer in Porous Media. Taylor\&Francis, New York.

Hu, J. and Xiao, J. (1995). New method for analysis of active magnetic regenerator in magnetic refrigeration at room temperature. Cryogenics, 35:10-104.

Jacobs, S. (2009). Modeling and optimal design of a multiplayer active magnetic refrigeration system. In Egolf, P. W., editor, Third International Conference on Magnetic Refrigeration at Room Temperature. International Institute of Refrigeration.

Jacobsson, P. and Sundqvist, B. (1989). Thermal conductivity and electrical resistivity of gadolinium as functions of pressure and temperature. Physical Review B, 40(14):9541-9551.

Janda, D., DeGregoria, A. J., Johnson, J., and Kral, S. (1989). Design of 
an active magnetic regenerative hydrogen liquefier. Advances in Cryogenic Engineering, 37:891-898.

Jeffreson, C. P. (1972). Prediction of breakthrough curves in packed beds. American Institute of Chemical Engineers, 18(2):409-20.

Johnson, J. W. and Zimm, C. B. (1996). Performance modeling of a 4 k active magnetic regenerative refrigerator. Journal of Applied Physics, 79:21712175 .

Joseph, R. and Schloemann, E. (1965). Demagnetizing field in nonellipsoidal bodies. Journal of Applied Physics, 36(5):1579-1593.

Kaviany, M. (1995). Principles of Heat Transfer in Porous Media. Springer, New York, 2nd edition.

Kawanami, T., Chiba, K., Sakurai, K., and Ikegawa, M. (2006). Optimization of a magnetic refrigerator at room temperature for air cooling systems. International Journal of Refrigeration, 29(8):1294-1301.

Kirol, L. D. and Mills, J. I. (1984). Numerical analysis of thermomagnetic generators. Journal of Applied Physics, 56(3):824-828.

Kitanovski, A. and Egolf, P. (2009). Application of magnetic refrigeration and its assessment. Journal of Magnetism and Magnetic Materials, $321(7): 777-781$.

Kitanovski, A., Egolf, P. W., Gendre, F., Sari, O., and Besson, C. (2005). A rotary heat exchanger magnetic refrigerator. In Egolf, P. W., editor, First International Conference on Magnetic Refrigeration at Room Temperature, 
International Institute of Refrigeration, pages 297-307. International Institute of Refrigeration.

Kuz'min, M. D. (2007). Factors limiting the operation frequency of magnetic refrigerators. Applied Physics Letters, 90:251916 (3pp).

Legait, I., Kedous-Lebouc, A., and Rondot, L. (2009). Numerical simulation and analysis of the refrigerant bed behavior using fluent software. In Egolf, P. W., editor, Third International Conference on Magnetic Refrigeration at Room Temperature, pages 295-302. International Institute of Refrigeration.

Li, P., Gong, M., Yao, G., and Wu, J. (2006). A practical model for analysis of active magnetic regenerative refrigerators for room temperature applications. International Journal of Refrigeration, 29:1259-1266.

Liu, M. and Yu, B. (2010). Numerical investigations on internal temperature distribution and refrigeration performance of reciprocating active magnetic regenerator of room temperature magnetic refrigeration. International Journal of Refrigeration, Accepted.

Morrish, A. H. (1965). The Physical Priciples of Magnetism. John Wiley \& Sons, Inc.

Nemec, D. and Levec, J. (2005). Flow through packed bed reactors: 1. single-phase flow. Chemical Engineering Science, 60(24):6947-6957.

Nielsen, K. K., Bahl, C. R. H., Smith, A., Bjørk, R., Pryds, N., and Hattel, J. (2009a). Detailed numerical modeling of a linear parallel-plate active magnetic regenerator. International Journal of Refrigeration, 32(6):14781486. 
Nielsen, K. K., Bahl, C. R. H., Smith, A., Pryds, N., and Hattel, J. (2010). A comprehensive parameter study of an active magnetic regenerator using a 2d numerical model. International Journal of Refrigeration, 33(4):753-764.

Nielsen, K. K., Engelbrecht, K., Bahl, C. R. H., Smith, A., Pryds, N., and Hattel, J. (2009b). Numerical modeling of multi-material active magnetic regeneration. In 7th World Conference on Experimental Heat Transfer, Fluid Mechanics and Thermodynamics.

Nielsen, K. K., Pryds, N., Smith, A., Bahl, C. R. H., and Hattel, J. (2009c). 2dimensional numerical modeling of active magnetic regeneration. In Egolf, P., editor, Third International Conference on Magnetic Refrigeration at Room Temperature, pages 251-258. International Institute of Refrigeration.

Oliveira, P. A., Trevizoli, P., Jr., J. R. B., and Prata, A. T. (2009). Numercial analsysis of a reciprocating active magnetic regenerator - part I. In Egolf, P. W., editor, Third International Conference on Magnetic Refrigeration at Room Temperature, pages 283-288. International Institute of Refrigeration.

Palacios, E., Wang, G. F., Burriel, R., Provenzano, V., and Shull, R. D. (2010). Direct measurement of the magnetocaloric effect in $\mathrm{Gd}_{5} \mathrm{Si}_{2} \mathrm{Ge}_{1.9} \mathrm{Ga}_{0.1}$. Journal of Physics: Conference Series, 200(9):092011.

Pecharsky, V. K. and Gschneidner, K. (2006). Advanced magnetocaloric materials: What does the future hold? International Journal of Refrigeration, 29:1239-1249.

Peksoy, O. and Rowe, A. (2005). Demagnetizing effects in active magnetic regenerators. Journal of Magnetism and Magnetic Materials, 288:424-432. 
Petersen, T. F., Engelbrecht, K., Bahl, C. R. H., Elmegaard, B., Pryds, N., and Smith, A. (2008a). Comparison between a 1d and a 2d numerical model of an active magnetic regenerative refrigerator. Journal of Physics D: Applied Physics, 41(10):105002.

Petersen, T. F., Pryds, N., Smith, A., Hattel, J., Schmidt, H., and Knudsen, H. (2008b). Two-dimensional mathematical model of a reciprocating room-temperature active magnetic regenerator. International Journal of Refrigeration, 31:432-443.

Risser, M., Vasile, C., Engel, T., Keith, B., and Muller, C. (2010). Numerical simulation of magnetocaloric system behavior for an industrial application. International Journal of Refrigeration, 33(5):973-981.

Rowe, A. and Barclay, J. (2003). Ideal magnetocaloric effect for active magnetic regenerators. Journal of Applied Physics, 93(3):1672-1676.

Rowe, A. and Tura, A. (2006). Experimental investigation of a three-material layered active magnetic regenerator. International Journal of Refrigeration, 29:1286-1293.

Sarlah, A. (2008). Thermohydraulic properties of heat regenerators in magnetic refrigerators. PhD thesis, University of Ljubljana, Slovenia.

Sarlah, A. and Poredos, A. (2005). Regenerator for magnetic cooling in shape of honeycomb. In Egolf, P. W., editor, First International Conference on Magnetic Refrigeration at Room Temperature, International Institute of Refrigeration, pages 283-290. International Institute of Refrigeration. 
Sarlah, A. and Poredos, A. (2010). Dimensionless numerical model for simulation of active magnetic regenerator refrigerator. International Journal of Refrigeration, 33 (6):1061-1067.

Shah, R. K. and Sekulic, D. P. (2003). Fundamentals of Heat Exchanger Design. John Wiley \& Sons, Inc., Hoboken, New Jersey.

Shir, F., Della Torre, E., Bennett, L. H., Della Torre, E., Bennett, L. H., Shull, R. D., and Mavriplis, C. (2004). Modeling of magnetization and demagnetization in magnetic regenerative refrigeration. IEEE Transactions on Magnetics, 40(4):2098-2100.

Shir, F., Yanik, L., Bennet, L. H., Torre, E. D., and Shull, R. D. (2003). Room temperature active regenerative magnetic refrigeration: Magnetic nanocomposites. Journal of Applied Physics, 93(10):8295-8297.

Siddikov, B., Wade, B., and Schultz, D. (2005). Numerical simulation of the active magnetic regenerator. Computers and Mathematics with Applications, 49:1525-1538.

Smailli, A. and Chahine, R. (1998). Thermodynamic investigations of optimum active magnetic regenerators. Cryogenics, 38:247-252.

Smith, A., Nielsen, K. K., Christensen, D. V., Bahl, C. R. H., Bjørk, R., and Hattel, J. (2010). The demagnetizing field of a nonuniform rectangular prism. Journal of Applied Physics, 107(10):103910.

Tagliafico, G., Scarpa, F., and Canepa, F. (2010). A dynamic 1-d model for a reciprocating active magnetic regenerator; influence of the main working parameters. International Journal of Refrigeration, 33(2):286-293. 
Tocado, L., Palacios, E., and Burriel, R. (2009). Entropy determinations and magnetocaloric parameters in systems with first-order transitions: Study of MnAs. Journal of Applied Physics, 105(9):093918.

Tusek, J., Sarla, A., Zupan, S., Prebil, I., Kitanovski, A., and Poredos, A. (2010a). A numerical optimization of a packed bed AMR. In Egolf, P. W., editor, Fourth International Conference on Magnetic Refrigeration at Room Temperature. International Institute of Refrigeration.

Tusek, J., Zupan, S., Sarlah, A., Prebil, I., and Poredos;, A. (2010b). Development of a rotary magnetic refrigerator. International Journal of Refrigeration, 33(2):294-300.

Willmott, A. J. (1964). Digital computer simulation of a thermal regenerator. International Journal of Heat and Mass Transfer, 7(11):1291-1302.

Yan, Z. and Chen, J. (1991). The characteristics of polytropic magnetic refrigeration cycles. Journal of Applied Physics, 70(4):1911-1914.

Yan, Z. and Chen, J. (1992). The effect of field-dependent heat capacity on the characteristics of the ferromagnetic Ericsson refrigeration cycle. Journal of Applied Physics, 72(1):1-5.

Yu, B., Gao, Q., Zhang, B., Meng, X., and Chen, Z. (2003). Review on research of room temperature magnetic refrigeration. International Journal of Refrigeration, 26:622-636.

Yu, B., Liu, M., Egolf., P. W., and Kitanovski, A. (2010). A review of magnetic refrigerator and heat pump prototypes built before the year 2010 . International Journal of Refrigeration, 33(6):1029-1060. 
Zhang, L., Sherif, S. A., DeGregoria, A. J., Zimm, C. B., and Veziroglu, T. N. (2000). Design and optimization of a 0.1 ton/day active magnetic regenerative hydrogen liquifier. Cryogenics, 40:269-278.

Zhang, L., Sherif, S. A., Veziroglu, T. N., and Sheffield, J. W. (1993). Second law analysis of active magnetic regenerative hydrogen liquefier. Cryogenics, 33(7):667-674. 


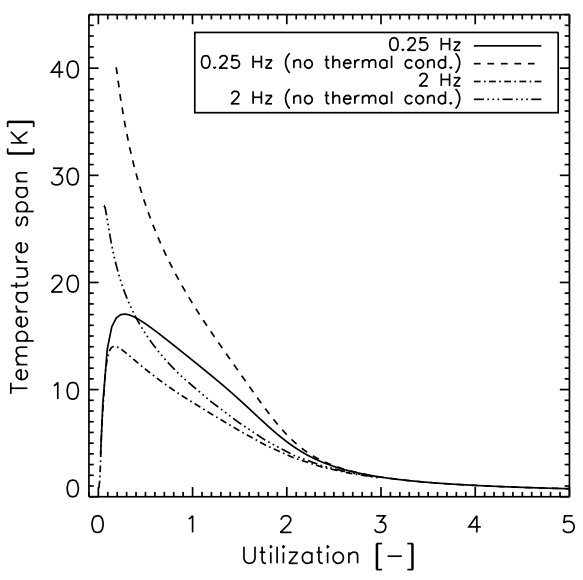

Figure A.1: The impact of the longitudinal thermal conduction on the predicted temperature span of the AMR at two different operating frequencies. The operating conditions in this case were an ambient temperature of $293 \mathrm{~K}$ and a regenerator of packed spheres with a diameter of $1 \mathrm{~mm}$. The model is published in Tusek et al. (2010a).

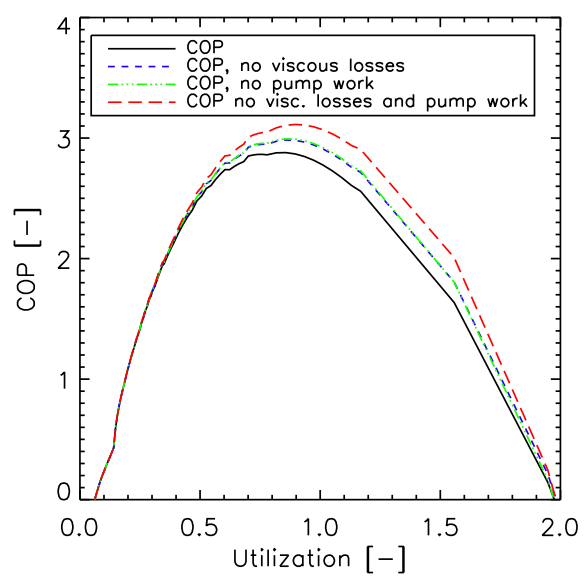

Figure A.2: The impact of the viscous losses on the COP of a packed spheres-based AMR. The model configuration was the same as that used for the results in Fig. A.1. The hot and cold side temperatures were set to 296 and $290 \mathrm{~K}$, respectively. 


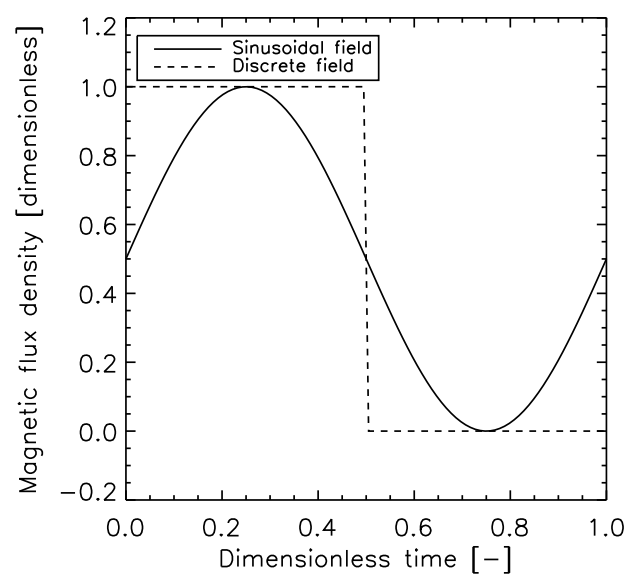

Figure A.4: Example of discrete on-off and continuous changing magnetic fields. 


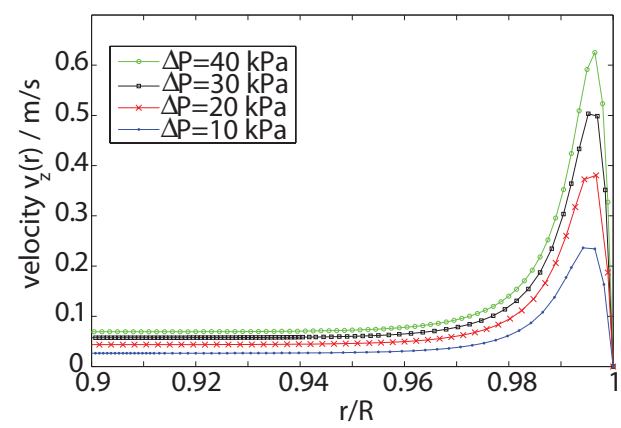

Figure A.5: Radial velocity distribution with $d_{r}=3 \mathrm{~cm}, d_{p}=1 \mathrm{~mm}, d_{r} / d_{p}=30$, regenerator length $L=7 \mathrm{~cm}$

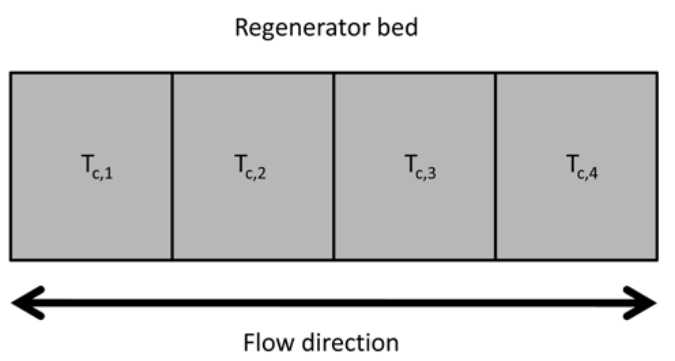

Figure A.6: Schematic of a layered regenerator. This case shows four different MCMs each with a specific Curie temperature denoted on the drawing. It is as yet not fully understood whether the optimum is an equal amount of each material, as shown here, or if the distribution of the materials should be asymmetric. 

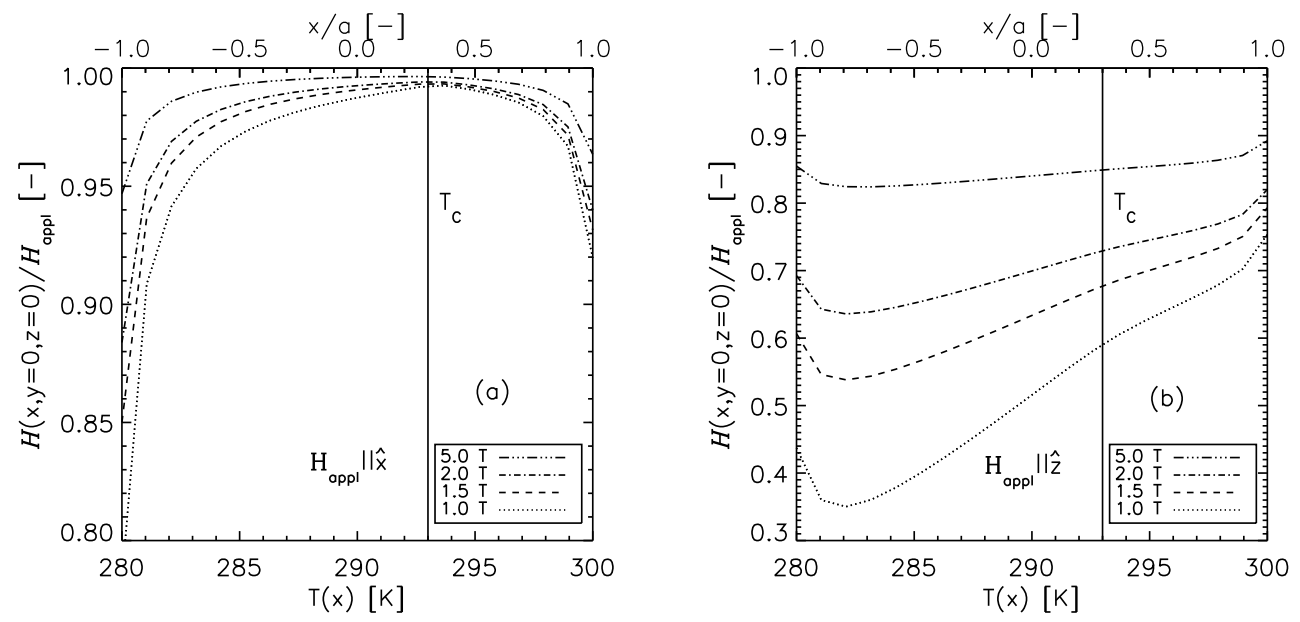

Figure A.7: Example of the internal magnetic field in a single-material magnetocaloric flat plate. A linear temperature profile is imposed from the cold end $(280 \mathrm{~K})$ to the hot end $(300 \mathrm{~K})$ and the internal magnetic field is calculated using the model from Smith et al. (2010). Left: the applied field is along the $x$-direction, i.e. the direction of the flow. Right: the applied field is along the $z$-direction, which is perpendicular to the flat plate. Four different applied fields are considered and the resulting internal magnetic field is plotted along the $x$-direction normalized to the applied field. The material used is Gd with a Curie temperature of $293 \mathrm{~K}$ (indicated on the figures). Reproduced from Smith et al. (2010). 


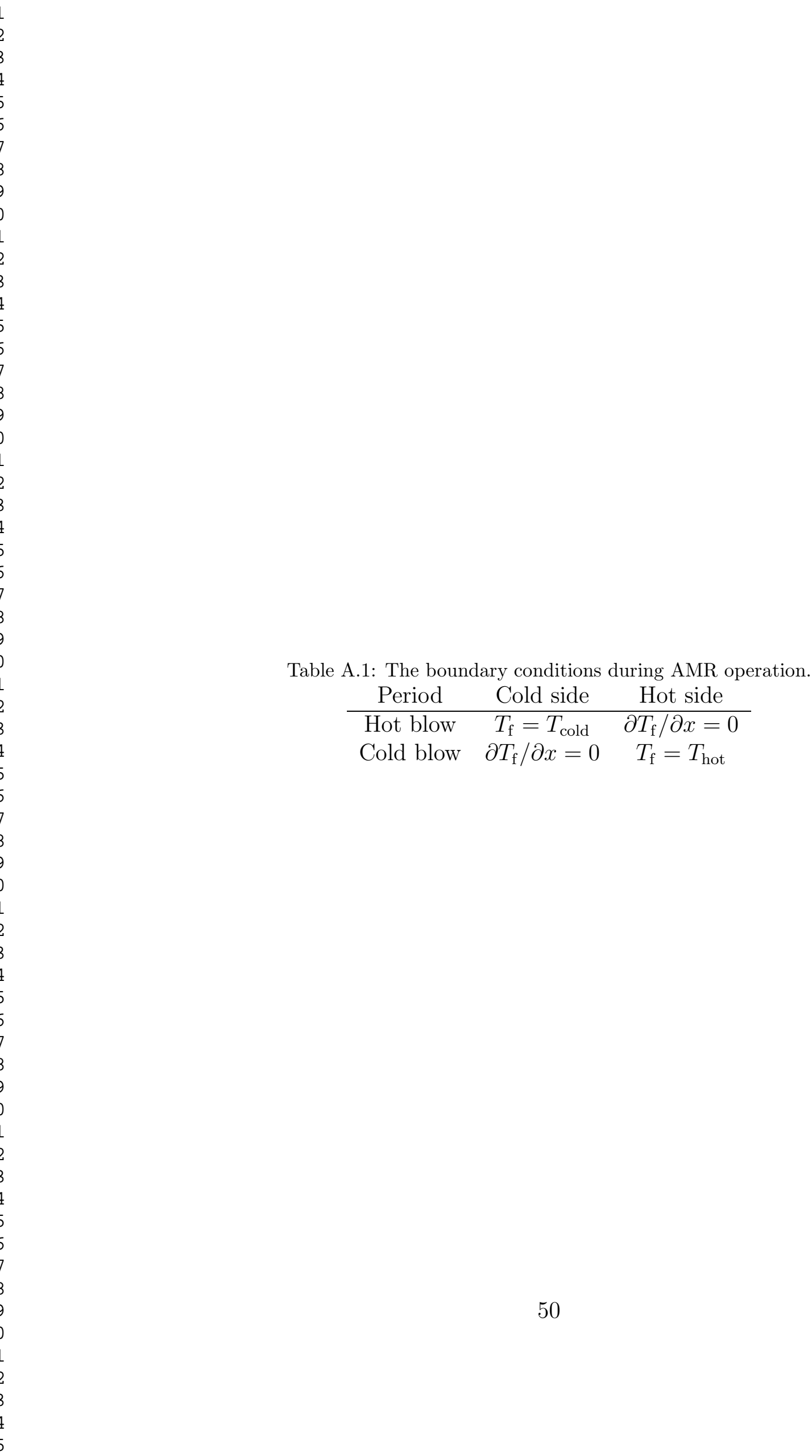




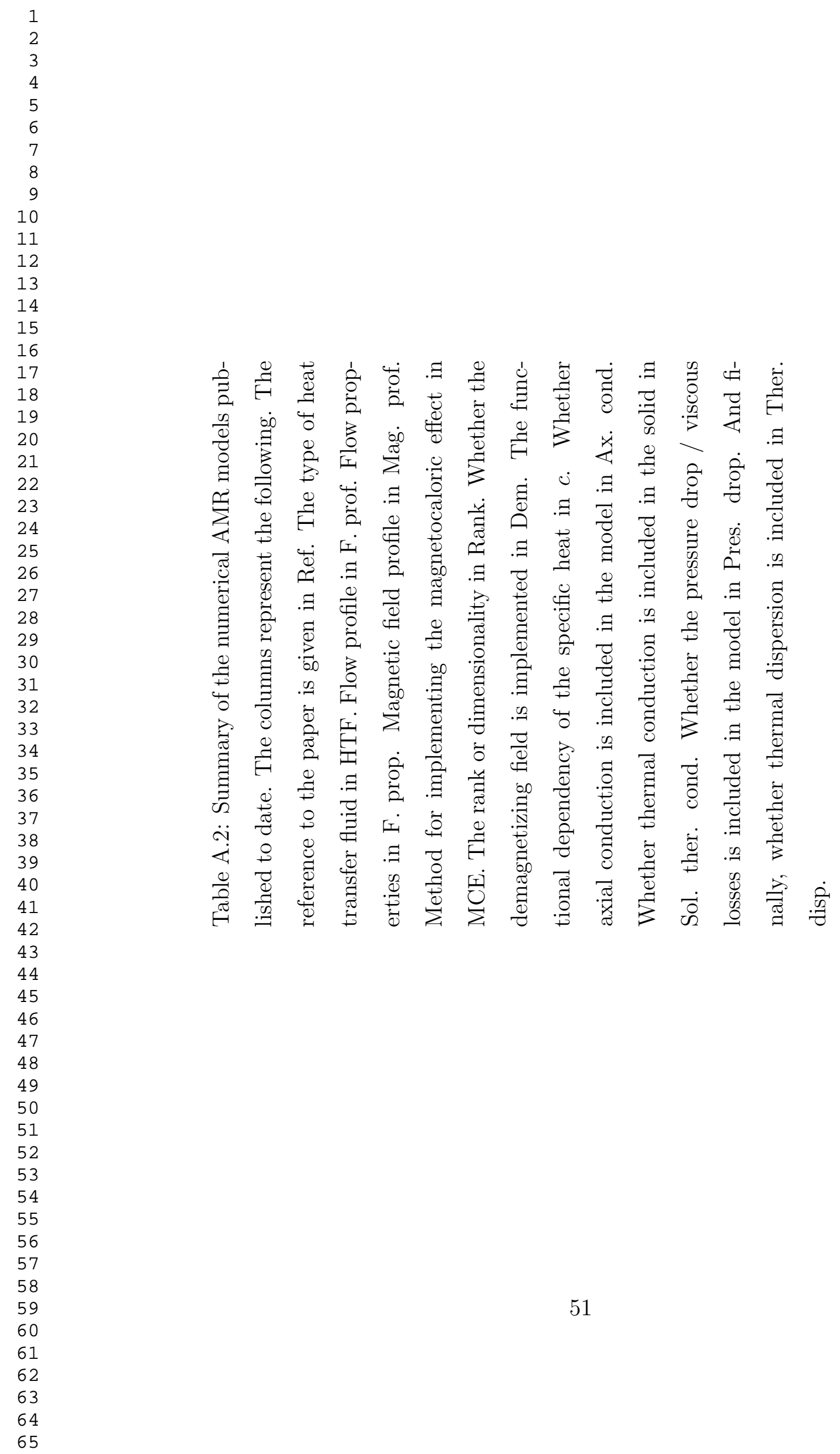




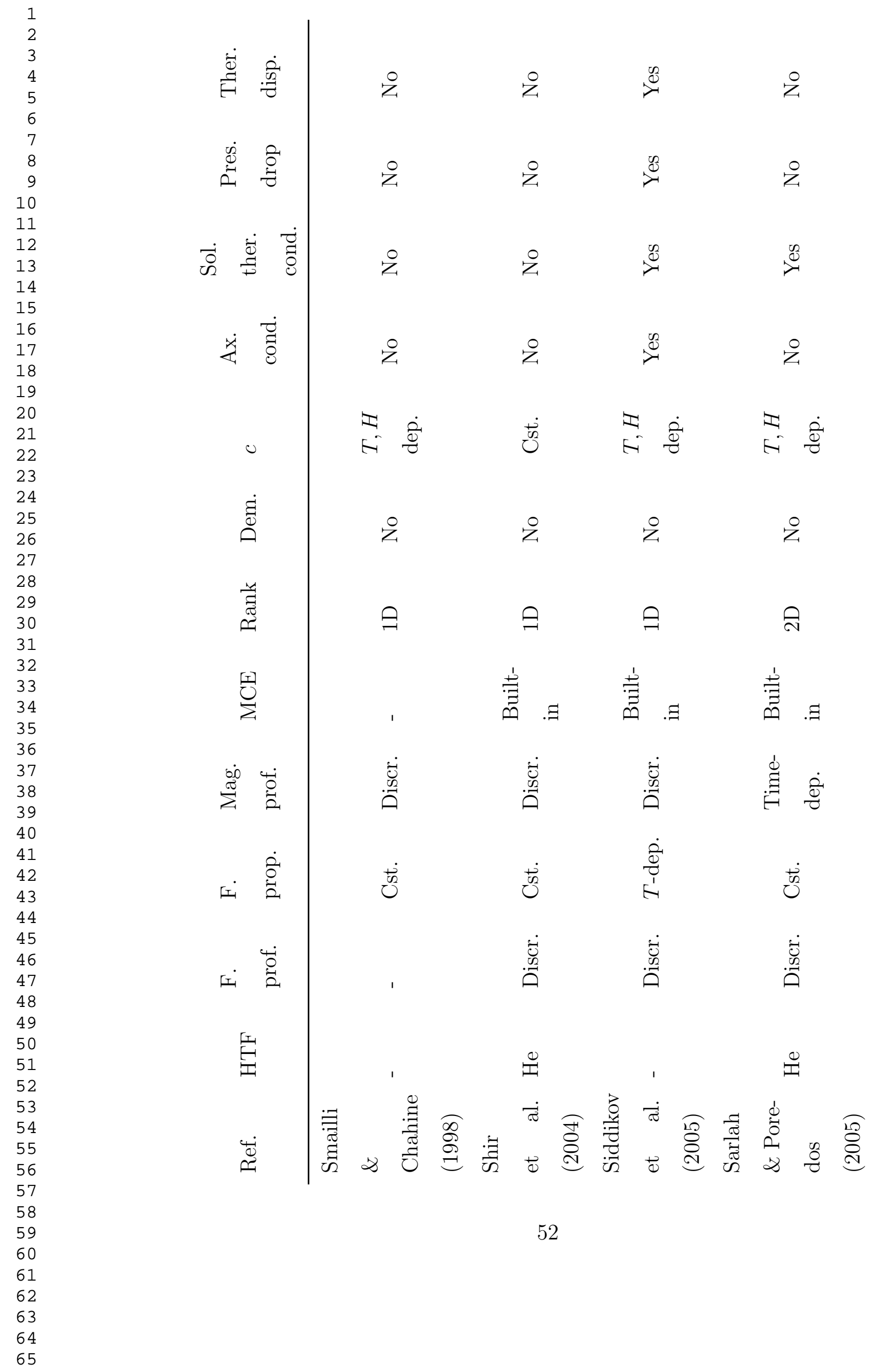




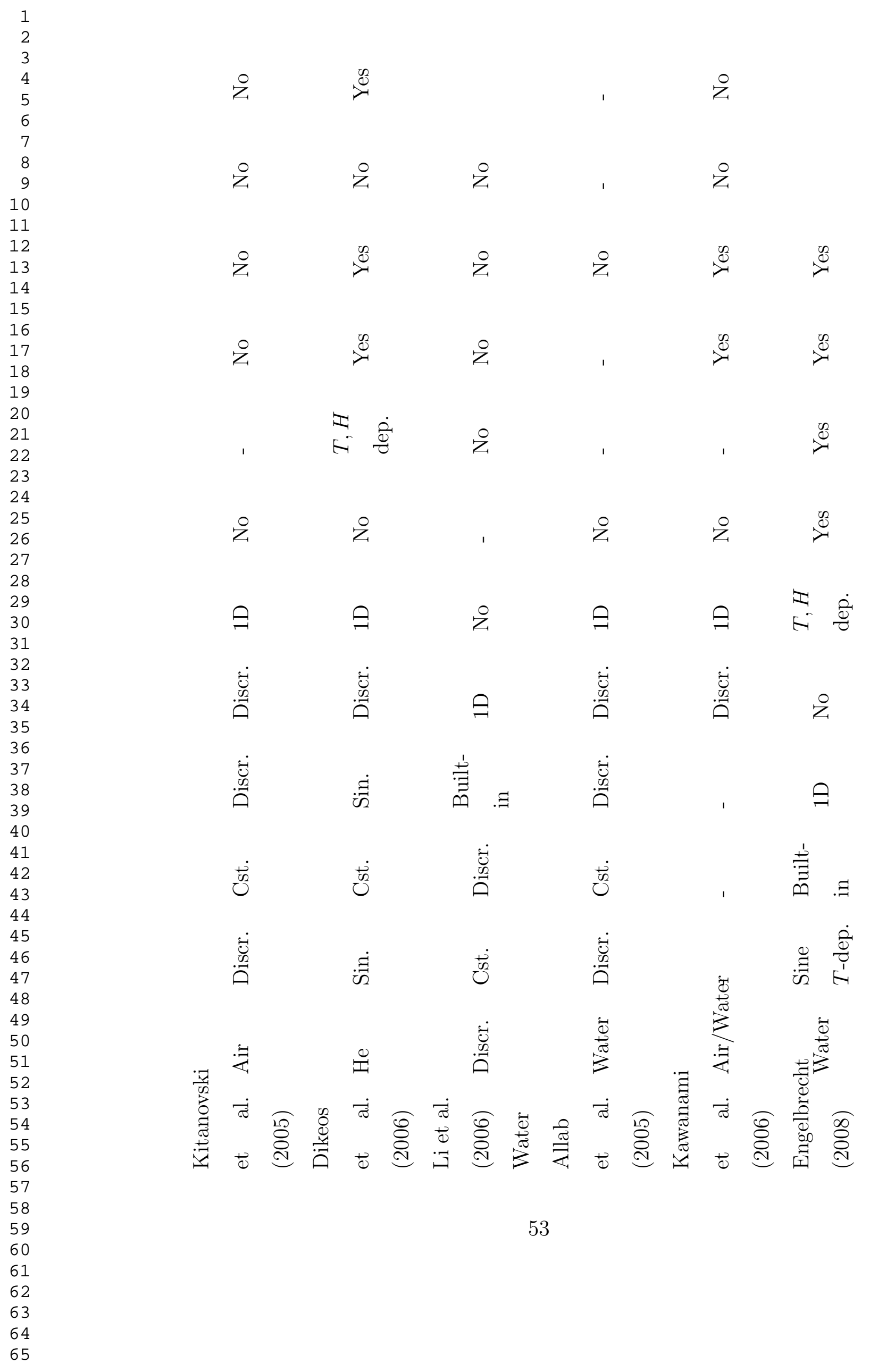




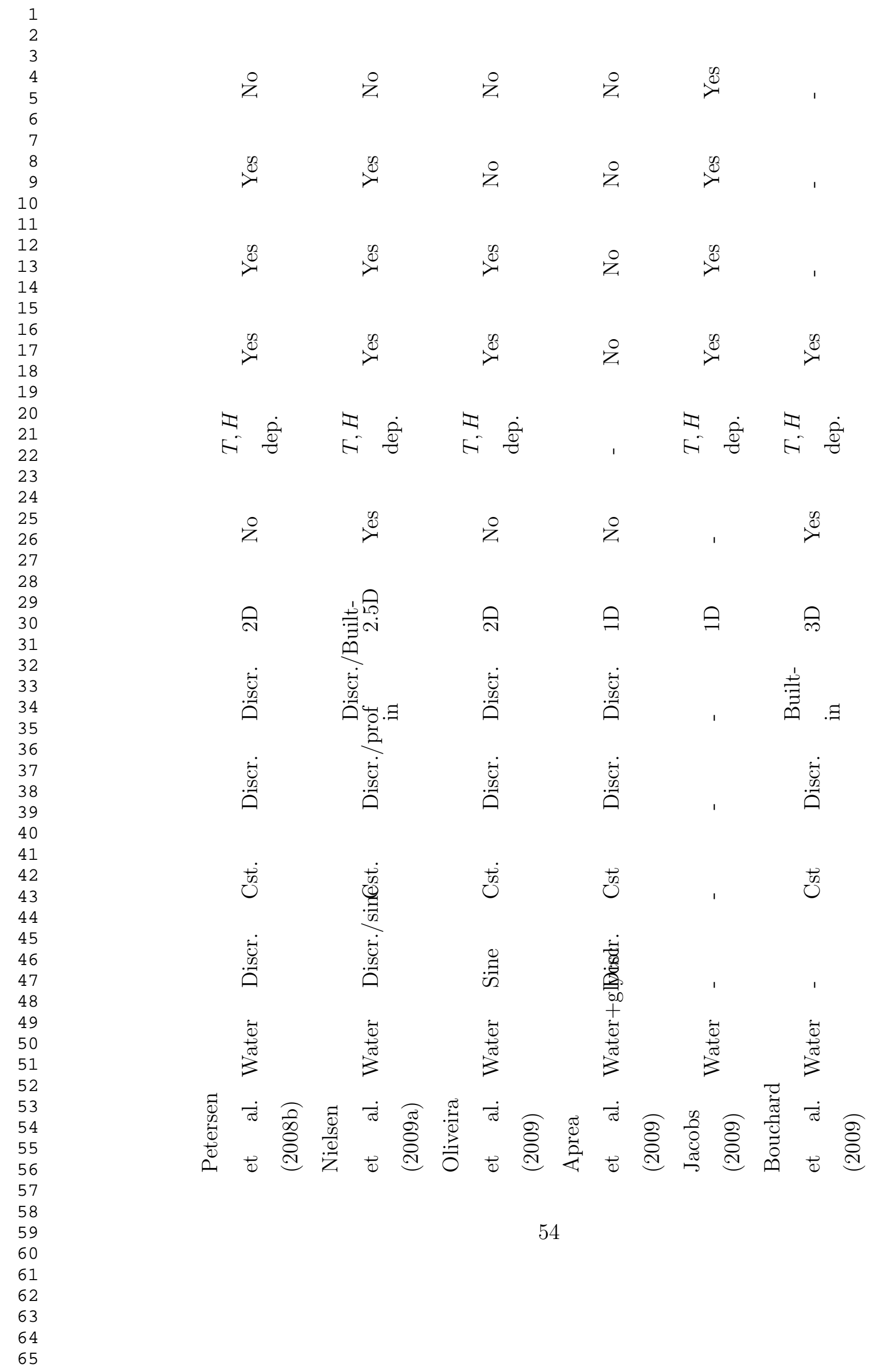




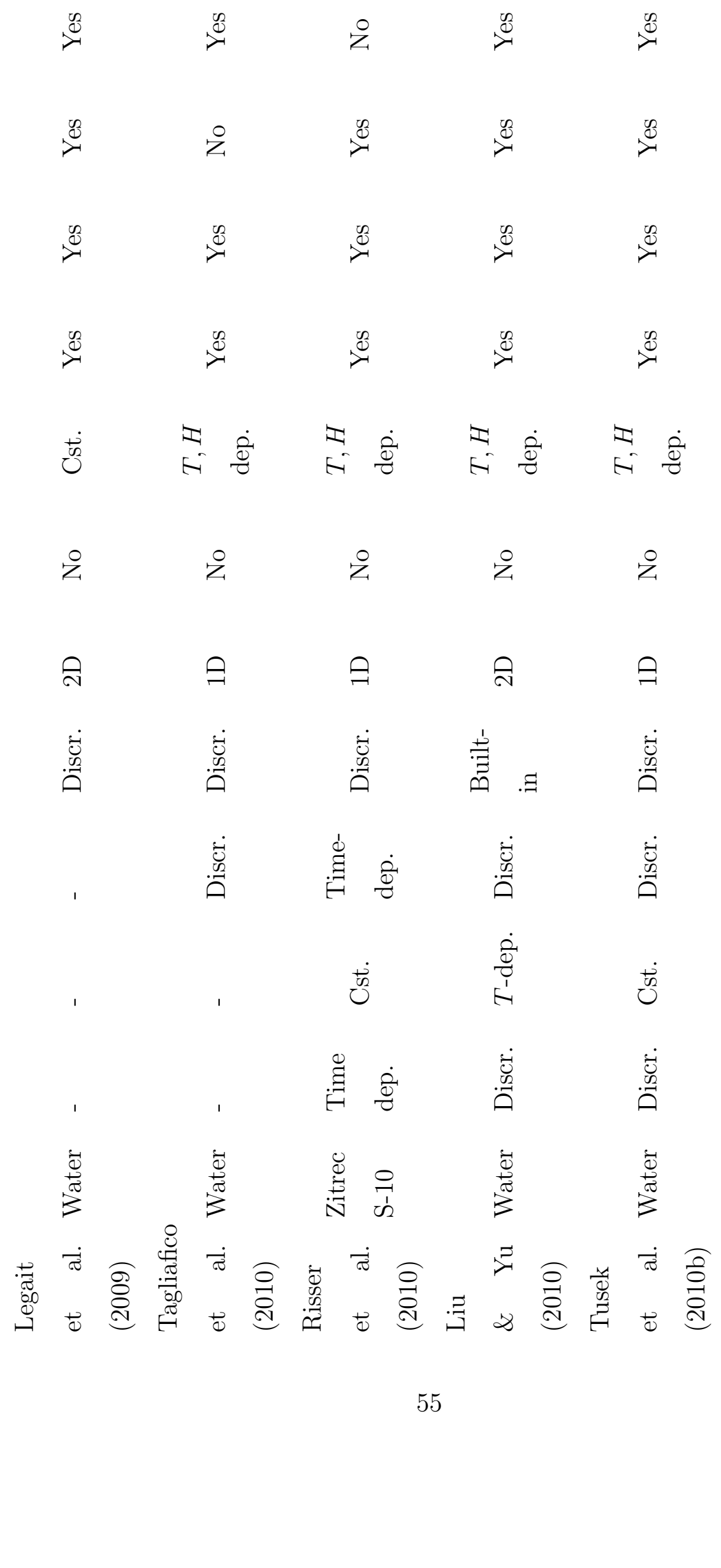


Felix Ziegler

Editor,

International Journal of Refrigeration

9 December 2010

Dear Dr. Ziegler,

The attached manuscript "Review on numerical modeling of active magnetic regenerators for room temperature applications", JIJR-D-10-00230 has been revised according to the reviewer's comments. We are most grateful for the comments and we believe they have contributed to improving the manuscript. In the following the changes are outlined in detail. We corrected minor errors in the manuscript and added a few new references as well.

The authors are: Kaspar K. Nielsen ${ }^{1,2}$, Jaka Tusek ${ }^{3}$, Kurt Engelbrecht ${ }^{2}$, Sandro Schopfer ${ }^{4}$, Andrej Kitanovski ${ }^{3}$, Christian Bahl ${ }^{2}$, Anders Smith ${ }^{2}$, Nini Pryds ${ }^{2}$, Alojz Poredos ${ }^{3}$.

${ }^{1}$ Department of Mechanical Engineering, Technical University of Denmark

${ }^{2}$ Fuel Cells and Solid State Chemistry Division, Ris $\varnothing$ DTU, Technical University of Denmark

${ }^{3}$ Faculty of Mechanical Engineering, University of Ljubljana, Slovenia

${ }^{4}$ Energy Systems Group, Department of Mechanical Engineering, University of Victoria, BC, Canada

Best regards,

Kaspar K. Nielsen (on behalf of the authors) 
Detailed response to the reviewer's comments.

- $\quad 2.1$ No statement about the length scale over which eq. (1) holds is made. Clearly this is assumed to hold over the regenerator length scale, i.e. one should clearly distinguish between intrinsic thermal conductivity and effective thermal conductivity (depends on geometrical configuration). Also 2D or 3D models can easily include Nusselt number correlations when the energy balance is treated as continuum that holds over the regenerator length. This requires feasible information of porosity and its gradient.

These points have been addressed with explicitly stating the validity of the assumed equations and that 2 and 3D models may apply Nu-Re correlations as this is, of course, correct. We have furthermore written that the general heat transfer equation of the solid (Eq. 1) includes the intrinsic heat transfer of the system.

- $\quad 2.9$ Dispersion is a macroscopic phenomena it arises from volume averaging of NSF equations. Of course this depends on the geometry. But in the first place it depends on the length scale over which the problem is considered. l.e. parallel plates can have high dispersion if the NSF equations are averaged over a repetitive domain.

The text has been updated accordingly. We do agree with the reviewer and we also believe that our description of the dispersion and longitudinal thermal conduction is sound.

- Conclusion: Statement "... it is obvious that each component should be implemented as detailed as possible..." is not necessarily true. A fully resolved physics model would be computational intensive and difficult to validate. They have their place; however, simplified models can be informative in that they can be tested and interepreted with ease.

We do agree and have updated the manuscript accordingly.

- It's too bad the review doesn't include all AMR modeling (high temp and low temp.) There are some informative papers at lower temperatures.

Yes, this is true. However, the title does explicitly state "room temperature applications". The length of the manuscript is already considerable and we believe our supposed expertise to be in the realm of room temperature applications. 
Responses to Technical Check Results

Felix Ziegler

Editor,

International Journal of Refrigeration

6 July 2010

Dear Dr. Ziegler,

These are our responses to the technical check results for the manuscript "Review on numerical modeling of active magnetic regenerators for room temperature applications".

1) Keywords provided should be selected from the given list

(http://www.elsevier.com/framework_products/promis_misc/30436keywords.pdf).

This has been addressed accordingly; one keyword was not on the list and has thus been removed.

2) Style for the unit should be $\mathrm{m} / \mathrm{s}-1$ and not $\mathrm{m} / \mathrm{s}$.

This has been changed accordingly

3) Vertical lines should not be used in tables.

These have been removed from the tables.

4) The text layout should be in double line spacing.

This has been changed such the "review" is used in the documentclass rather than "preprint" in complete accordance with the Elsevier tex template.

Best regards,

Kaspar K. Nielsen (on behalf of the authors) 